\title{
Etude des spectres de diffusion Raman des cyanures de sodium et de potassium en phase plastique
}

\author{
D. Fontaine et R. M. Pick \\ Département de Recherches Physiques (*), Université P.-et-M.-Curie, 4, place Jussieu, 75230 Paris Cedex 05, France
}

(Reçu le 21 mars 1979, révisé le 9 juillet 1979, accepté le 16 juillet 1979)

\begin{abstract}
Résumé. - Les trois spectres Raman indépendants de basse fréquence de $\mathrm{NaCN}$ ont été obtenus en phase plastique I entre 300 et $400 \mathrm{~K}$. Ils sont interprétés à l'aide d'un modèle dans lequel la diffusion de la lumière est uniquement due aux changements d'orientation des ions cyanures. Le spectre de type $F_{2 \mathrm{~g}}$ s'explique comme une libration autour des directions $[1,0,0]$, et l'on discute l'origine de la très grande largeur observée. Un modèle de réorientation, par effondrement de la barrière de potentiel, entre directions $[1,0,0]$ équivalentes, explique quantitativement le spectre $\mathrm{E}_{\mathrm{g}}$ aux diverses températures. Les spectres correspondants de $\mathrm{KCN}$ en phase plastique $\mathrm{I}$ montrent le même type de comportement, mais ne permettent qu'une interprétation qualitative, le minimum principal du potentiel orientationnel n'étant sans doute pas dans la direction $[1,0,0]$.
\end{abstract}

\begin{abstract}
The three independent Raman spectra of sodium cyanide have been obtained, in the plastic phase (phase I), between 300 and $400 \mathrm{~K}$. They are interpreted by using a model in which the scattering mechanism is only attributed to changes of the cyanide ion orientations. The $F_{2 g}$ spectrum is explained as due to librations around $[1,0,0]$ directions and the origin of its very large width is discussed. A reorientation model between $[1,0,0]$ equivalent directions in which a breakdown of the potential barrier is involved, explains quantitatively the $\mathbf{E}_{\mathbf{g}}$ spectrum at different temperatures. The corresponding spectra of potassium cyanide in the plastic phase, show the same behaviour, but do not allow a qualitative interpretation to be obtained, the principal minimum of the orientational potential being probably not in the $[1,0,0]$ direction.
\end{abstract}

1. Introduction. - La phase plastique des cyanures de sodium et de potassium (aussi appelée phase I) s'étend respectivement de $283,7 \mathrm{~K}$ et $168,3 \mathrm{~K}$ à la température de fusion $(836,8 \mathrm{~K}$ et $907,6 \mathrm{~K})$. L'étude de l'orientation et des mouvements des ions peut être effectuée grâce à diverses techniques expérimentales. A cet égard, les plus intéressantes sont la résonance magnétique nucléaire $[1,2]$, la diffusion inélastique de neutrons $[3,4,5]$, la diffusion Raman [6-13] ainsi que l'étude de la transition entre la phase I et la phase II de plus basse température $[14,15]$.

Dans le cas de la diffusion Raman, les difficultés expérimentales (mauvaise polarisation des spectres) n'avaient pas permis, jusqu'à présent, une interprétation satisfaisante des résultats. L'amélioration de la technologie nous a permis d'obtenir des spectres de bonne qualité sur une large gamme de température (cf. 4.3). Nous montrerons (cf. 4.3) qu'un modèle simple de diffusion Raman et de réorientation des ions cyanures permet :

(*) Laboratoire associé au C.N.R.S., $\mathbf{n}^{\circ} 71$.
- de mesurer l'anisotropie de la fonction de probabilité d'orientation des ions cyanures (cf. 5.5),

- d'expliquer les spectres de basses fréquences des deux cyanures (cf. 6 et 7) et de montrer l'existence de librations assez bien définies dans ces deux corps.

Avant d'entreprendre cette étude, nous allons commencer par rappeler les résultats statiques et dynamiques déjà obtenus sur ces deux cristaux.

2. Rappels sur les résultats de la diffusion élastique et inélastique. - 2.1 Diffusion ÉLASTIQUE. - L'interprétation des spectres de diffraction de rayons $\mathrm{X}$ montre que la structure des deux cyanures étudiés est cubique faces centrées avec une unité formulaire par maille primitive [16] pour leur phase I. Une telle structure est incompatible avec la symétrie propre de l'ion cyanure $\left(\mathrm{C}_{\infty}^{\mathrm{v}}\right)$. Diverses hypothèses ont été formulées afin d'interpréter ce résultat allant de la rotation libre [17], à un jeu d'orientations équivalentes [18]. Ce sont, en fait, des expériences de diffraction de neutrons $[3,4]$ qui ont permis de faire progresser le problème, en mesurant avec précision 
les deux premiers coefficients significatifs de la fonction de probabilité $P(\theta, \varphi)$ de trouver un ion $\mathrm{CN}^{-}$, dont les coordonnées par rapport aux axes du cristal soient $\theta$ et $\varphi$. Quoique la troncature de $P(\theta, \varphi)$ à ses deux termes ne permette pas de fixer avec certitude l'allure de cette fonction, les auteurs ont proposé (Fig. 1) que celle-ci présentait pour le cyanure de sodium un maximum dans la direction $(1,0,0)$, un maximum relatif dans la direction $(1,1,1)$ et un minimum dans la direction $(1,1,0)$; dans le cas du cyanure de potassium, le maximum était dans la direction $(1,1,1) ;(1,0,0)$ est une direction du maximum relatif et le minimum est toujours dans la direction $(1,1,0)$. Dans ce dernier cas, des mesures faites à $296 \mathrm{~K}$ et $180 \mathrm{~K}$ ne montrent qu'une très faible évolution des résultats, les traits généraux n'étant pas profondément modifiés.

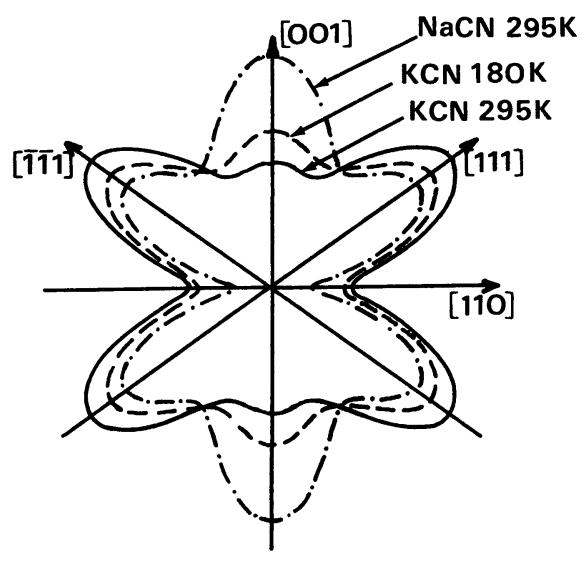

Fig. 1. - Probabilité $P(\theta, \varphi)$ de trouver un ion $\mathrm{CN}^{-}$dans la direction angulaire $(\theta, \varphi)$, d'après [4].

[Probability of finding a cyanide ion in the $(\theta, \varphi)$ angular direction, after [4].]

2.2 Diffusion INÉlastiQue. - Dans la phase I des cristaux de cyanure de sodium et de potassium, la symétrie de translation n'est conservée que pour les cations et les centres de masse des ions cyanures. Depuis 1970, on sait (cf. par exemple [19, 20]) que dans les expériences de diffusion (de la lumière ou des neutrons), le désordre d'orientation intervient à deux niveaux. D'une part, les modes propres du système ne sont plus, en général, des ondes planes; d'autre part, la conservation du quasi-moment n'est plus réalisée (et en particulier la règle de sélection $q=0$ n'est plus vraie en diffusion Raman). Les expériences de diffraction neutroniques [22, 25] ont confirmé ce point de vue. En effet, en dehors des cas des faibles transferts d'énergie et de quasi-moment, la fonction de réponse mesurée est très large et ne permet que de déterminer approximativement que la densité des états des excitations élémentaires. Celle-ci s'étend, en gros, jusqu'à $200 \mathrm{~cm}^{-1}$ pour $\mathrm{KCN}$ et $250 \mathrm{~cm}^{-1}$ pour $\mathrm{NaCN}$. L'absence de relief dans les spectres obtenus (et en particulier l'absence de librations marquées) est attribuée par les auteurs, à la très faible durée de vie des phonons, à cause des interactions et des réorientations de grands angles. Nous verrons dans la partie 6.3, que nous ne partageons pas entièrement ce point de vue.

Les modes acoustiques restent, par contre, assez bien visibles, surtout près du centre de zone. La comparaison entre les résultats de la diffusion Brillouin [14] et de la propagation ultrasonore [23] met en évidence, pour les phonons se propageant dans les plans $(1,0,0)$ et polarisés suivant $(1,1,0)$, deux régimes différents. La transition entre ceux-ci se faisant pour (voir figure 2) :

$$
\begin{array}{llll}
\mathrm{KCN} & T=169 \mathrm{~K}\left(T_{\mathrm{c}}+1 \mathrm{~K}\right) & \sigma \neq 2 \mathrm{~cm}^{-1} & q \# 0,1, \\
\mathrm{NaCN} & T=293 \mathrm{~K}\left(T_{\mathrm{c}}+10 \mathrm{~K}\right) & \sigma \neq 6 \mathrm{~cm}^{-1} & q \# 0,2 .
\end{array}
$$

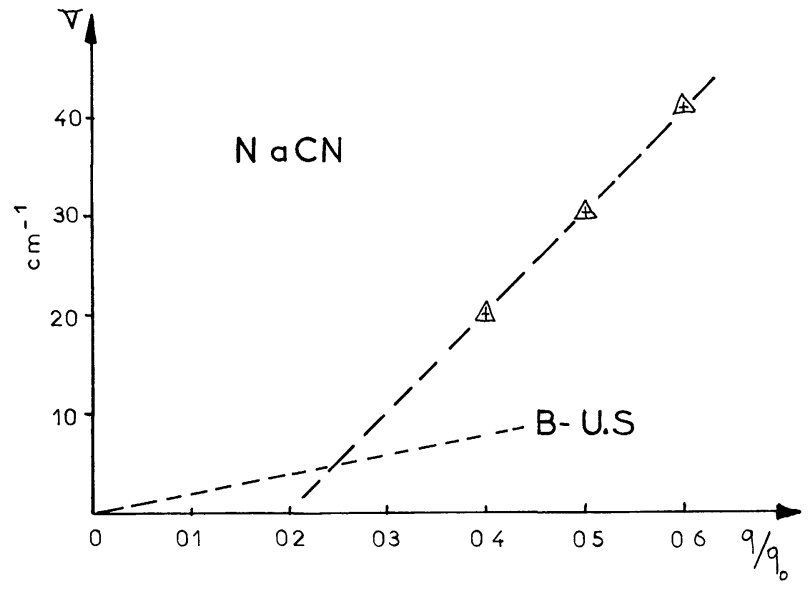

Fig. 2. - Branche acoustique (direction $\Gamma X$ ) des courbes de dispersions dans $\mathrm{NaCN}$. ------ Courbe extrapolée des mesures de diffusion Brillouin et de propagation ultrasonore [14]; $A$ Valeurs expérimentales de diffusion inélastique de neutrons [21].

[Acoustic phonon branch ( $\Gamma X$ direction) for $\mathrm{NaCN}$. ------ Curve extrapolated from Brillouin scattering and ultrasonic propagation [14]; A Experimental values from neutron inelastic scattering [21].]

L'interprétation de ces deux régimes a été faite précédemment $[14,24]$ et est basée sur l'idée que, pour ces phonons, le régime de haute fréquence est uniquement lié aux mouvements de translations des ions cyanures et alcalins tandis qu'en basse fréquence, les translations se couplent, entre autres, aux fluctuations d'orientations des cyanures. Ce phénomène s'accentue lorsque la température baisse et conduit à une transition de phase vers la phase II.

3. Méthodes expérimentales. - 3.1 PrÉPARATION ET ORIENTATION DES CRISTAUX. - Les cristaux ont été préparés par la méthode de Bridgman. Par suite de la faible valeur de la constante élastique $c_{44}$ et de son évolution thermique, de nombreuses contraintes 
apparaissent lors du refroidissement des cristaux, produisant des biréfringences artificielles qui modifient profondément les états de polarisation de la lumière diffusée. L'orientation des cristaux par taille et polissage risquant d'aggraver ces défauts, nous n'avons utilisé qu'une découpe par clivage qui fournit des plans parallèles aux directions $(1,0,0)$ de la maille cubique.

3.2 Le spectromètre utilisé est un triple monochromateur T800 de la société CODERG qui possède un très bon taux de réjection de la lumière parasite. Sauf indication contraire, les largeurs à mi-hauteur des fonctions d'appareil utilisées sont de $2 \mathrm{~cm}^{-1}$, ce qui est suffisant en basse fréquence.

3.3 Montages EXPÉRIMENTAuX. - Les montages utilisés sont directement liés à l'orientation et à la symétrie des cristaux. Les trois spectres $\mathrm{A}_{1 \mathrm{~g}}, \mathrm{E}_{\mathrm{g}}$ et $\mathrm{F}_{2 \mathrm{~g}}$ linéairement indépendants sont obtenus à partir de 4 combinaisons linéaires de ceux-ci, à savoir $(X, Y, Z / /(1,0,0))$ :

$$
\begin{array}{ll}
\mathrm{S}_{1}=\mathrm{A}_{1 \mathrm{~g}}+4 \mathrm{E}_{\mathrm{g}} & X(Z Z) Y \\
\mathrm{~S}_{2}=\mathrm{F}_{2 \mathrm{~g}} & X(Z X) Y, \text { en diffusion trans- }
\end{array}
$$$$
\text { et }
$$$$
\mathrm{S}_{3 \mathrm{~g}}=\mathrm{A}_{1 \mathrm{~g}}+\mathrm{E}_{\mathrm{g}}+\mathrm{F}_{2 \mathrm{~g}} \quad X(Z+Y, Z+Y) \bar{X}
$$$$
\mathrm{S}_{4 \mathrm{~g}}=3 \mathrm{E}_{\mathrm{g}} \quad X(Z+Y, Z-Y) \bar{X} \text {, en }
$$
rétrodiffusion .

3.4 Problèmes eXPÉRIMENTAuX. - 3.4.1 Comptabilité des mesures. - A partir des quatre spectres expérimentaux précédents, on peut définir un invariant : $H=S_{1}+S_{2}=S_{3}+S_{4}=A_{1 g}+4 E_{g}+F_{2 g}$, entre les deux montages, ce qui permet, en principe, de déterminer un facteur d'échelle. Expérimentalement, nous avons constaté que cette relation n'était pas vérifiée et nous avons pu montrer que les défauts provenaient essentiellement d'une intensité parasite située en dessous de $60 \mathrm{~cm}^{-1}$ dans les spectres de rétrodiffusion polarisés et analysés verticalement. Il pourrait sembler étonnant que ceci soit observable dans un triple monochromateur, mais il faut se rappeler que dans un montage de rétrodiffusion, l'impact du faisceau laser est mis au point sur la fente d'entrée du spectromètre, la lumière incidente est donc beaucoup moins bien éliminée que dans les autres montages et nos expériences ont montré que ce type de défaut n'apparaissait ni en diffusion transverse, ni en rétrodiffusion pour une analyse et polarisation croisées. Le facteur d'échelle a alors été mesuré indépendamment en utilisant le mode d'élongation de l'ion cyanure dont la fréquence est située vers $2000 \mathrm{~cm}^{-1}$.

3.4.2 Effets dus à la biréfringence artificielle. Un autre problème expérimental est lié aux effets de biréfringence artificielle. Leur correction a été réalisée approximativement à l'aide de la méthode suivante [11].
Nous avons constaté que, dans de très bonnes conditions expérimentales (cristal immergé dans un milieu de même indice, cristaux fraîchement clivés, peu trempés), le taux de dépolarisation de la raie d'élongation pouvait n'atteindre que $0,8 \%$, en montage transverse aussi bien qu'en rétrodiffusion. Nous avions à notre disposition une raie de type $A_{1 \mathrm{~g}}$ (nous montrerons ultérieurement que ceci n'est vrai qu'en première approximation). Cette raie permet alors de mesurer les effets des biréfringences dans des cas où les conditions expérimentales sont plus mauvaises. On peut en effet noter que :

- les effets de biréfringence artificielle conservent la somme des intensités lumineuses,

- la diffusion Raman est un processus incohérent.

Par un calcul simple, utilisant les coefficients de dépolarisation de la raie d'élongation de l'ion $\mathrm{CN}^{-}$, à savoir :

$$
r=I_{\mathrm{VH}} / I_{\mathrm{vv}} \quad \text { et } \quad R=I_{\mathrm{HV}} / I_{\mathrm{Vv}},
$$

il est possible de déterminer les trois spectres corrigés : $A_{1 g}, E_{g}$ et $F_{2 g}$. Un exemple de la méthode utilisée est détaillée dans l'Annexe I. Signalons en outre que la raie d'élongation, après correction des effets de dépolarisation accidentelle, fournit un étalon d'intensité qui nous permet de comparer les intensités relatives des spectres de basses fréquences en fonction de la température (Fig. 13), compte tenu de l'hypothèse que l'intensité intégrée de cette raie ne varie pas avec la température.

3.4.3 Discussion de la méthode de correction. Par suite de l'existence du bruit de photon dans les spectres, cette méthode ne peut être acceptable que si les corrections apportées, aux spectres mesurés expérimentalement, sont faibles. Cette méthode ne peut donc suppléer ni à la préparation de cristaux de bonne qualité optique (clivage), ni à la recherche de régions contenant peu de contraintes (choix du domaine diffusant), ce qui peut parfois se révéler long et fastidieux. D'autre part, nous utilisons la raie d'élongation située à $2000 \mathrm{~cm}^{-1}$ environ de la raie excitatrice pour corriger nos spectres de basses fréquences. En fait, si la biréfringence artificielle possède une certaine dispersion en fréquence, celle-ci peut introduire une erreur systématique dans la valeur des coefficients notés : $r, R$. Cet effet sera minimisé si l'on prend soin de placer le pinceau lumineux excitateur le plus près possible de la face de sortie, en diffusion transverse.

Un calcul assez simple, basé sur un modèle de lame biréfringente, dont les axes optiques sont situés dans des directions $(1,1,0)$ permet d'évaluer la correction à apporter au coefficient $R$, qui est le seul affecté. On trouve que l'on a :

$$
R(\sigma=0) \# 0,8 R\left(\sigma \# 2000 \mathrm{~cm}^{-1}\right) \text {. }
$$

Le procédé décrit conduit à une surestimation de ce coefficient, mais celle-ci étant faible, il n'en a pas été tenu compte. 
3.5 Résultats. - A titre d'exemple, nous montrons pour $\mathrm{NaCN}$ à $295 \mathrm{~K}$ et $388 \mathrm{~K}$ (Figs. 3 et 4) un spectre $\mathrm{F}_{2 \mathrm{~g}}$ non corrigé et corrigé. On constate, dans les deux cas, que non seulement l'intensité mais aussi la forme des spectres peut être altérée par les effets de pollution. Sur la figure 5 sont reportés, à la même échelle, les spectres de type $A_{1 g}, E_{g}$ et $F_{2 g}$ de $\mathrm{NaCN}$. Compte tenu de la dispersion des résultats (due à la très grande intensité du spectre $\mathrm{E}_{\mathrm{g}}$ en très basse fréquence), on peut considérer que l'intensité du spectre $A_{1 \mathrm{~g}}$ est nulle au-dessus de $20 \mathrm{~cm}^{-1}$. Nous avons aussi retrouvé ce résultat pour $\mathrm{KCN}$ (Fig. 6) ; cependant, en dessous de $-60^{\circ} \mathrm{C}$, la dépolarisation des spectres est telle que nous n'avons pas pu confirmer ce résultat dans ce domaine de température.

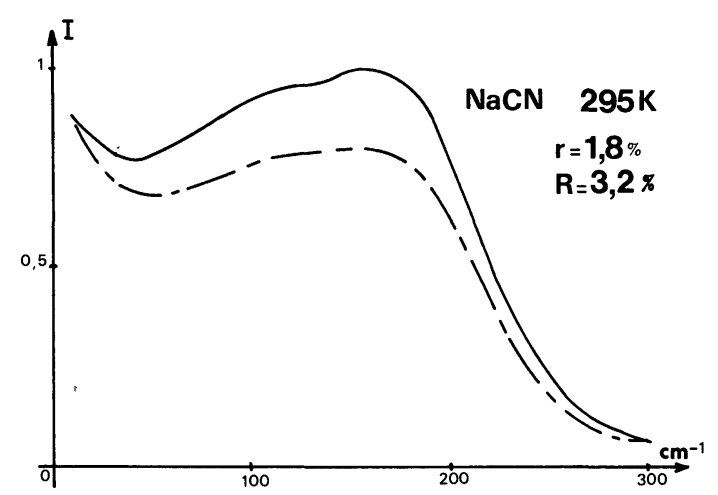

Fig. 3. - Exemple de correction d'un spectre de type $\mathrm{F}_{2 \mathrm{~g}}$ pour des coefficients $r$ et $R$ faibles. L'échelle des intensités est arbitraire. ----- Spectre expérimental; — Spectre corrigé.

[Typical correction for a $F_{2 g}$ spectrum for small correction coefficients $r$ and $R$. Intensity arbitrary units. ------ Experimental spectrum; - Corrected spectrum.]

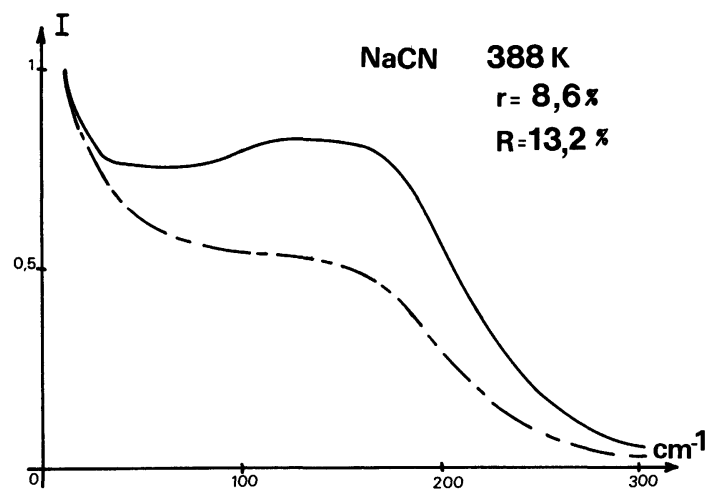

Fig. 4. - Exemple de correction d'un spectre de type $F_{2 \mathrm{~g}}$ pour des coefficients $r$ et $R$ relativement grands. L'échelle des intensités est arbitraire. On remarque la modification importante du spectre pour des fréquences inférieures à $100 \mathrm{~cm}^{-1}$. - - - Spectre expérimental; - Spectre corrigé.

[Typical correction for a $\mathrm{F}_{2 \mathrm{~g}}$ spectrum for larger correction coefficients $r$ and $R$. Intensity arbitrary units. Note the large modification of the spectrum below $100 \mathrm{~cm}^{-1}$. - - Experimental spectrum; - Corrected spectrum.]

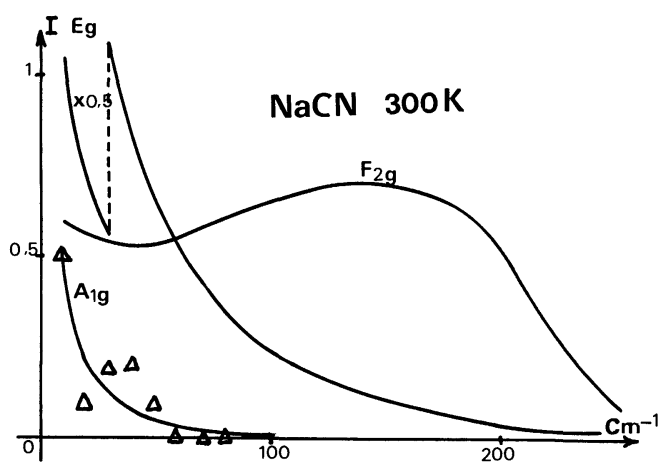

Fig. 5. - Spectre de diffusion Raman de $\mathrm{NaCN}$ de basse fréquence (même échelle d'intensité pour les trois spectres).

[Low frequency Raman scattering spectra of $\mathrm{NaCN}$ (same intensity scale for the three curves).]

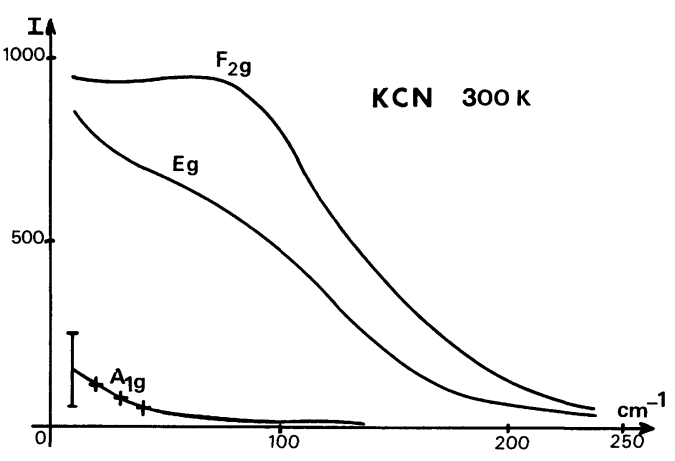

Fig. 6. - Spectre de diffusion Raman de $\mathrm{KCN}$ de basse fréquence (même échelle d'intensité pour les trois spectres).

[Low frequency Raman scattering spectrum of KCN (same intensity scale for the three curves).]

4. Origine de la diffusion Raman de basse fréquence. - 4.1 MODÈLE MICROSCOPIQUE DE RÉORIENTATION DES IONS CYANURES. - L'étude de nos spectres Raman, de leur évolution et des résultats de la diffusion inélastique de neutrons, nous a amené à proposer un modèle microscopique pour les réorientations dont nous allons ici préciser les origines et les caractéristiques.

Les mesures de diffraction $[3,4]$ montrent que l'encombrement stérique dans les cyanures de sodium et de potassium ne permet pas d'envisager des rotations libres des ions ni d'ailleurs de chemin de réorientation à barrière de potentiel relativement basse. De plus, les réorientations ne peuvent pas se faire par passage par dessus une barrière de potentiel due aux ions voisins puisque, du fait de l'encombrement stérique, celle-ci doit être pratiquement infinie. Cependant, la mesure des coefficients de Debye-Waller (voir tableau I), permet d'envisager des réorientations couplées aux déplacements des ions voisins, produisant ainsi des effondrements de la barrière de potentiel. Notre modèle est donc de type phonon assisted 
Tableau I. - Déplacements quadratiques moyens dans $\mathrm{KCN}$ et $\mathrm{NaCN}$ [4].

[Mean square displacements in $\mathrm{KCN}$ and $\mathrm{NaCN}$ [4].]

$\begin{array}{lccc} & \mathrm{KCN}(295 \mathrm{~K}) & \mathrm{KCN}(180 \mathrm{~K}) & \mathrm{NaCN}(295 \mathrm{~K}) \\ \left\langle u_{x}^{2}\right\rangle\left(\AA^{2}\right) \mathrm{M}^{+} & 0,0622[16] & 0,0660[16] & 0,0680[9] \\ \left\langle u_{x}^{2}\right\rangle\left(\AA^{2}\right) \mathrm{CN}^{-} & 0,0614[18] & 0,0578[16] & 0,0590[8]\end{array}$

reorientation, et conduit à des processus à anharmonicité très élevée dans lequel il serait vain de rechercher un couplage préférentiel avec un ou plusieurs modes particuliers de la première zone de Brillouin.

Schématiquement un tel modèle est caractérisé par deux durées de vie : une durée moyenne $T_{\mathrm{r}}$ de libration de l'ion cyanure autour d'une direction donnée et un temps $T_{\mathrm{R}}$, durée moyenne de réorientation de l'ion. Nous montrerons en 6.2 qu'il est possible de préciser des ordres de grandeurs de ces temps grâce à l'utilisation des spectres de diffusion.

4.2 Couplage AVeC LeS ONDES ÉleCtromagnétIQUES. - Le mécanisme de réorientation proposé ci-dessus ne sera observable en spectroscopie optique que s'il se couple avec la lumière. On sait que la diffusion Raman est produite par la modification (en fonction du temps) des tenseurs de polarisabilité diélectrique. Ce changement de polarisabilité local peut avoir trois causes :

a) Le changement d'orientation des ions cyanures (modes de pivotements). Ces molécules ayant un tenseur diélectrique anisotrope (il est diagonal dans les axes de l'ion) tout changement d'orientation de l'ion se traduit par un changement de polarisabilité dans les axes du cristal.

b) Le changement d'orientation de l'ion cyanure ou son déplacement par rapport à un ion métallique peut changer la polarisabilité de ce dernier. Un tel effet est certainement négligeable car les ions sodium et potassium sont très peu polarisables, ayant des couches électroniques complètes très stables.

c) Le changement d'orientation de l'ion cyanure ou son déplacement par rapport à un ion métallique peut enfin changer la polarisabilité de l'ion $\mathrm{CN}^{-}$ dans ses propres axes. Ce dernier effet pourrait être d'un ordre de grandeur comparable à l'effet $a$ ). L'analyse des expériences nous montrera qu'elles sont en fait complètement interprétables par l'effet $a$ ), si bien que nous pourrons négliger l'effet $c$ ). C'est ce point de vue que nous adopterons ici.

Par ailleurs, dans la structure $\mathrm{NaCl}$ du cristal moyen, il n'existe aucune diffusion due à un processus harmonique, puisque chaque ion est centre de symétrie. Les spectres de basse fréquence que nous avons mesurés sont donc dus :

- soit à un processus à deux phonons,

- soit à la levée de la règle de sélections $(q \# 0)$ à cause du désordre.

LE JOURNAL DE PHysiQue. - T. 40, No 11 , NOVembre 1979
Dans notre cas, il est facile d'éliminer l'existence d'un spectre à deux phonons de basse fréquence puisque aucun spectre n'est observé au-delà de $250 \mathrm{~cm}^{-1}$, alors que les résultats de diffusion inélastique de neutrons suggèrent que le spectre de phonons s'étend jusqu'à cette fréquence approximativement. On s'attend donc à un spectre de second ordre allant jusqu'à environ $500 \mathrm{~cm}^{-1}$, ce qui n'est pas observé. Le spectre de basse fréquence est donc dû au désordre orientationnel des ions cyanures.

4. 3 Equations DE LA DIFFUSION ET JUSTIFICATION DU MODÈLE DE DIFFUSION. - Dans l'hypothèse du cristal moléculaire, l'intensité diffusée, à la fréquence $\omega$, s'écrit [26] :

$$
S=\sum_{i j k l} n_{i} n_{k} I_{i j k l}(\omega) E_{j} E_{l}^{*}
$$

où $n_{i}$ est une composante du vecteur unitaire de la direction d'analyse de la lumière diffusée et $E_{j}$ une composante du vecteur champ électrique incident, avec :

$$
I_{i j k l}(\omega)=\text { T.F. }\left\langle\sum_{\mathbf{R} \mathbf{R}^{\prime}} \varepsilon_{i j}^{\mathrm{R}^{*}}(t) \varepsilon_{k l}^{\mathbf{R}^{\prime}}(0) \exp i \mathbf{q} \cdot\left(\mathbf{R}-\mathbf{R}^{\prime}\right)\right\rangle .
$$

Dans cette formule, $\mathbf{q}$, le vecteur d'onde de l'onde diffusée est toujours très inférieur à la longueur d'un vecteur du réseau réciproque et par conséquent, le terme de phase peut être pris égal à l'unité. D'autre part, les tenseurs sont relatifs à un ion $\mathrm{R}$ à l'instant $t$ : on constate l'existence d'un couplage entre les positions des différents ions (les crochets représentent ici une moyenne thermodynamique prise sur les états initiaux du système).

Si la diffusion Raman n'est produite que par les mouvements angulaires des ions cyanures et si $\varepsilon_{0}$ est le tenseur de polarisabilité diélectrique d'un ion cyanure dans ses propres axes, on peut remplacer, dans (2), $\varepsilon^{\mathrm{R}}(t)$ par

$$
\varepsilon(\Omega)=R^{-1}(\Omega) \varepsilon_{0} R(\Omega)
$$

où $R(\Omega)$ est la matrice de rotation d'angle $\Omega$ et $\Omega(t)$ représente la direction de l'ion cyanure à l'instant $t$.

Comme $\varepsilon_{0}$ à la symétrie $D_{\infty \mathrm{h}}$, en posant

$$
\Gamma=R^{-1}(\Omega) \mathbf{M} R(\Omega)
$$

avec

$$
\begin{gathered}
M=\left(\begin{array}{rrr}
1 & 0 & 0 \\
0 & 1 & 0 \\
0 & 0 & -2
\end{array}\right), \\
\alpha=\frac{1}{3}\left(2 \varepsilon_{x x}+\varepsilon_{z z}\right) \quad \text { et } \quad \beta=\frac{\varepsilon_{x x}-\varepsilon_{z z}}{3},
\end{gathered}
$$

on en déduit

$$
I_{i j k l} \sim \beta^{2} \text { T.F. }\left\langle\sum_{\mathrm{RR}^{\prime}} \Gamma_{i j}\left(\Omega^{\mathrm{R}}(t)\right) \Gamma_{k l}\left(\Omega^{\mathrm{R}^{\prime}}(0)\right)\right\rangle .
$$


Les cristaux de cyanures de sodium et de potassium étant de symétrie cubique faces centrées, les valeurs des intensités des différents spectres habituellement mesurés peuvent alors s'exprimer comme :

$$
\begin{aligned}
\mathrm{A}_{1 \mathrm{~g}} & \sim \alpha^{2} \delta(\omega) \\
\mathrm{E}_{\mathrm{g}} & \sim \frac{\beta^{2}}{4} \text { T.F. } \sum_{\mathbf{R R}^{\prime}}\left\langle\Gamma_{i i}\left(\Omega^{\mathrm{R}^{\prime}}(t)\right) \Gamma_{j j}\left(\Omega^{\mathrm{R}}(0)\right)\right\rangle \\
\mathrm{F}_{2 \mathrm{~g}} & \sim \beta^{2} \text { T.F. } \sum_{\mathbf{R R}^{\prime}}\left\langle\Gamma_{i j}\left(\Omega^{\mathrm{R}^{\prime}}(t)\right) \Gamma_{i j}\left(\Omega^{\mathrm{R}}(0)\right)\right\rangle .
\end{aligned}
$$

L'expression (4) montre que l'hypothèse d'une diffusion produite uniquement par les mouvements angulaires des ions cyanures conduit à un spectre de type $A_{1 \mathrm{~g}}$ sans changement de fréquence. Les spectres de $\mathrm{NaCN}$ et de $\mathrm{KCN}$ (Figs. 5, 6), faits à diverses températures (respectivement entre $300 \mathrm{~K}$ et $450 \mathrm{~K}$ et $210 \mathrm{~K}$ et $450 \mathrm{~K}$ ) montrent qu'il en est bien ainsi $\left(^{1}\right)$ (les limitations dans l'échelle des températures sont dues à une très forte dépolarisation des spectres lors de l'approche de la température de transition $(\mathrm{KCN})$ ). On pourra donc utiliser le modèle proposé pour la diffusion Raman, sachant que tous les autres processus diffusionnels sont, de fait, d'intensités beaucoup plus faibles que celui décrit dans notre modèle.

5. Application du modèle de diffusion Raman à la raie d'élongation. - 5.1 POSITION DU PROBLÈME. La raie d'élongation de l'ion cyanure est observée à $2088,6 \mathrm{~cm}^{-1}$ pour le cyanure de sodium $(T=300 \mathrm{~K})$ et à $2075,3 \mathrm{~cm}^{-1}$ pour le cyanure de potassium (même température). A priori, cette raie peut être de symétrie quelconque. Cependant, nous avons dit précédemment qu'elle apparaissait pratiquement comme une raie de type $A_{1 \mathrm{~g}}$; nous étudierons plus précisément cette affirmation ultérieurement et verrons que ceci n'est vrai qu'en première approximation. Il est plus juste de dire que la seule raie fine qui apparaisse est de type $A_{1 \mathrm{~g}}$. La largeur de cette raie doit d'abord être discutée puisque l'on constate, en général, dans les cristaux moléculaires désordonnés, des raies larges (de 6 à $15 \mathrm{~cm}^{-1}$ ) pour des modes actifs en infrarouge alors que les figures 7 et 8 montrent que, dans notre cas, cette largeur est de l'ordre de $2 \mathrm{~cm}^{-1}$. La largeur est, en général, expliquée en considérant chaque molécule comme un oscillateur indépendant faiblement perturbé par les autres molécules. La largeur provient alors, et de la durée de vie propre de l'oscillateur indépendant (la molécule qui diffuse est dans un état excité dont

( $\left.{ }^{1}\right)$ Durant cette rédaction, nous avons pu consulter la thèse de L. W. Winchester [6] sur les cyanures de sodium et de potassium. Bien que ce dernier n'ait pratiqué aucune correction sur les spectres et que son cristal présente des effets de dépolarisation certains $(10 \%)$ (notamment observables sur le mode d'élongation), il confirme approximativement ce résultat expérimental.

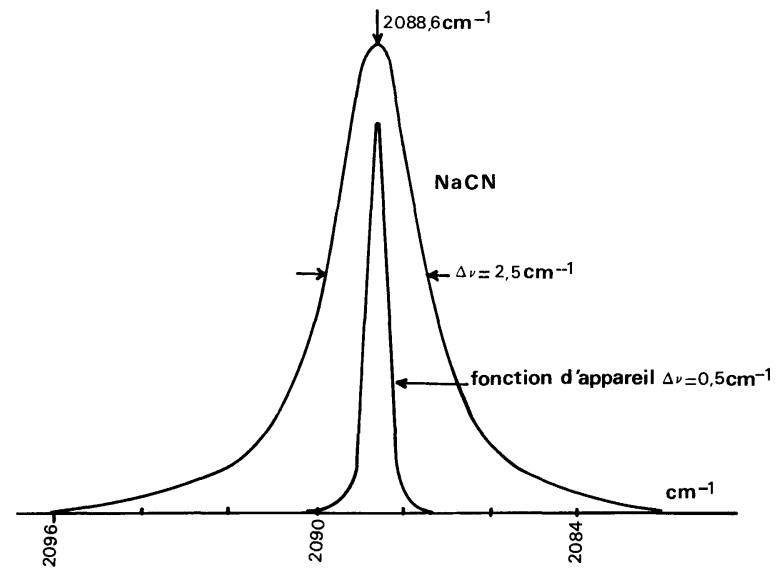

Fig. 7. - Spectre de haute fréquence de type $A_{1 g}+4 E_{g}$ de $\mathrm{NaCN}$ $(T=300 \mathrm{~K})$.

[High frequency Raman scattering spectrum of $\mathrm{NaCN}\left(\mathrm{A}_{1 \mathrm{~g}}+4 \mathrm{E}_{\mathrm{g}}\right.$ type, $T=300 \mathrm{~K})$.]

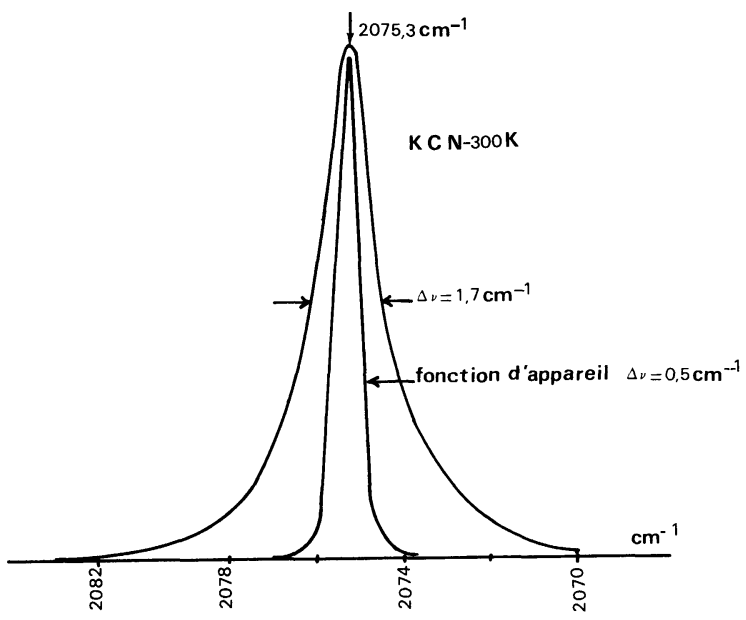

Fig. 8. - Spectre de haute fréquence de type $A_{1 g}+4 E_{g}$ de $K C N$ $(T=300 \mathrm{~K})$.

[High frequency Raman scattering spectrum of $\mathrm{KCN}\left(\mathrm{A}_{1 \mathrm{~g}}+4 \mathrm{E}_{\mathrm{g}}\right.$ type, $T=300 \mathrm{~K})$.]

le temps de vie est fini), et de la variation de la fréquence de vibration sous l'influence des perturbations (effets de champ local). Cet effet peut être statique (le champ local est différent pour diverses molécules, mais sa valeur ne change pas pendant le temps de vie de l'état excité) ou dynamique (le champ local varie de façon appréciable pendant ce temps de vie). Dans ces conditions, une raie de largeur très faible ne peut s'expliquer que par une très grande durée de vie et par un faible effet de champ local. Cette faiblesse peut avoir, en fait, deux origines différentes : soit que le champ local varie suffisamment vite pour qu'il ait un effet nul sur la vibration (c'est le rétrécissement par le mouvement), soit qu'il soit lui-même faible. La période de la vibration d'élongation étant de $1,7 \times 10^{-2} \mathrm{ps}$, le premier effet semble assez 
improbable. Nous allons montrer que c'est la deuxième explication qui est correcte, ce qui implique, a posteriori, que les élongations des divers ions ne sont pas corrélées. Un ordre de grandeur de l'effet de champ local peut être obtenu assez simplement (voir par exemple [29]). La grande difficulté est l'évaluation de la dérivée du moment dipolaire par rapport à la coordonnée, $Q$, qui sera majorée par

$$
\left(\frac{\partial p}{\partial Q}\right)<1 \mathrm{D} / \AA \text {. }
$$

On en déduit aisément que, compte tenu d'un effet de moyenne dû aux diverses orientations des ions, l'effet de champ local produit un élargissement en tout état de cause inférieur à $1 \mathrm{~cm}^{-1}$. On en déduit que, pour ce mode d'élongation, les effets de corrélation entre les vibrations internes des diverses molécules pourront être négligés.

5.2 INTENSITÉS DIFFUSÉES. - En utilisant un raisonnement identique à celui fait précédemment, on peut exprimer sans difficultés les différents spectres diffusés relatifs au mode d'élongation. Dans les formules donnant l'intensité (4), (5), (6), la polarisabilité diélectrique sera simplement remplacée par le produit de la polarisabilité dérivée ( $\alpha^{\prime}$ et $\beta^{\prime}$ ) par la coordonnée normale du mode $(Q)$. D'autre part, l'effet de découplage entre les mouvements internes des ions conduit à la disparition de la sommation sur les atomes différents ( $R$ et $R^{\prime}$ ) qui apparaissent dans les formules précédentes et l'on obtient :

$$
\begin{gathered}
\mathrm{A}_{1 \mathrm{~g}} \sim \alpha^{\prime 2} \delta\left(\omega-\omega_{\mathrm{V}}\right) \otimes \sum_{\mathrm{R}}\left\langle Q^{\mathrm{R}^{2}}\right\rangle \\
\mathrm{E}_{\mathrm{g}} \sim \sum_{\mathrm{R}} \frac{\beta^{\prime 2}}{4} \delta\left(\omega-\omega_{\mathrm{V}}\right) \otimes \mathrm{T} . \mathrm{F} .\{ \\
\left.\quad\left\langle Q^{\mathrm{R}^{2}} \Gamma_{x x}\left(\Omega^{\mathrm{R}}(t)\right) \Gamma_{x x}\left(\Omega^{\mathrm{R}}(0)\right)\right\rangle\right\} \\
\mathrm{F}_{2 \mathrm{~g}} \sim \sum \beta^{\prime 2} \delta\left(\omega-\omega_{\mathrm{V}}\right) \otimes \mathrm{T} . \mathrm{F} .\{ \\
\left.\left\langle Q^{\mathrm{R}^{2}} \Gamma_{x x}\left(\Omega^{\mathrm{R}}(t)\right) \Gamma_{x y}\left(\Omega^{\mathrm{R}}(0)\right)\right\rangle\right\} .
\end{gathered}
$$

Nous constatons que le spectre de type $A_{1 \mathrm{~g}}$ n'est pas affecté par les mouvements angulaires, tandis que ceux de type $E_{g}$ et $F_{2 g}$ le sont. D'autre part, les spectres de haute fréquence dépendent de la fonction d'autocorrélation d'orientation des ions cyanures, tandis que les spectres de basse fréquence dépendent des fonctions de corrélation. L'ensemble des spectres est donc nécessaire à la compréhension des mouvements angulaires des ions et est non redondant.

5.3 RÉSUltATS EXPÉRIMENTAUX. - La figure 9 montre un spectre de type $\mathrm{E}_{\mathrm{g}}$ obtenu en rétrodiffusion sur un monocristal de cyanure de potassium. On constate par la hauteur du bruit de fond que, malgré une radiation excitatrice très intense $(1,5 \mathrm{~W})$, ce spectre est de très faible intensité, ce qui rend très difficile des mesures quantitatives. Ce résultat expérimental justifie l'affirmation faite précédemment que le spectre de la raie d'élongation est pratiquement de

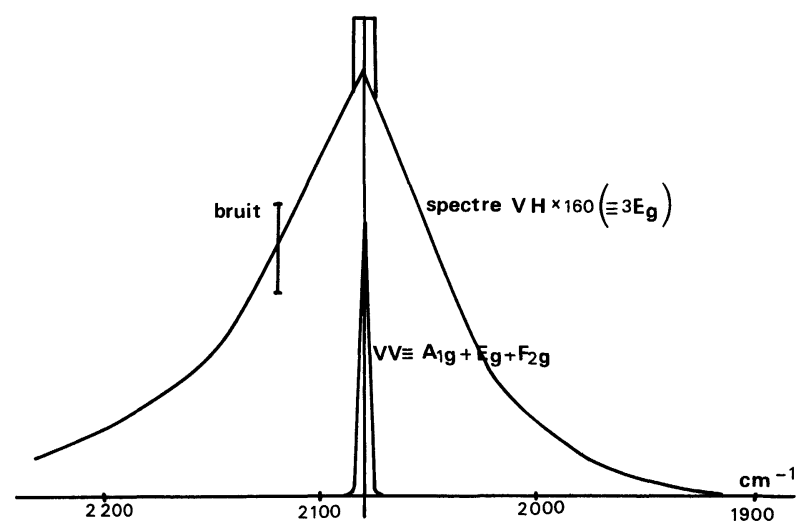

Fig. 9. - Exemple de spectre de type $\mathrm{E}_{\mathrm{g}}$, pour KCN. Largeur de la fonction d'appareil $: 3,5 \mathrm{~cm}^{-1}$. Puissance de la raie excitatrice $(\lambda=5145 \AA)=1,5 \mathrm{~W}$.

LExample of an $\mathrm{E}_{\mathrm{g}}$ type spectrum of $\mathrm{KCN}$. Slit $=3.5 \mathrm{~cm}^{-1}$, power of the excitating line $(\lambda=5145 \AA)=1.5 \mathrm{~W}$.]

type $A_{1 g}$, ce qui nous a permis de définir un estimateur de défauts de polarisation (cf. 3.4.2).

5.4 DÉFINITION ET MESURE DES CORRÉLATIONS ET DES ORIENTATIONS. - En l'absence de corrélations statiques et dynamiques entre les ions cyanures, les spectres de haute fréquence et de basse fréquence devraient être identiques.

Il est donc possible de mettre en évidence celles-ci par comparaison directe des spectres, soit de type $\mathrm{E}_{\mathrm{g}}$, soit de type $\mathrm{F}_{2 \mathrm{~g}}$. Dans cette partie, nous utiliserons une autre méthode en exploitant seulement les résultats des mesures d'intensité intégrées des spectres qui fournissent des informations sur les probabilités d'orientation et leurs corrélations. Nous commencerons par montrer que le spectre intégré des spectres de haute fréquence permet de déterminer certains termes du développement de la fonction de probabilité d'orientation des ions cyanures. Le même calcul effectué sur les spectres de basse fréquence nous permettra de définir quantitativement l'effet des corrélations dynamiques et statiques.

5.4.1 Calcul du premier coefficient anisotrope de la fonction $P(\theta, \varphi)$. - En utilisant les formules (8) et (9), on obtient :

$$
\begin{aligned}
& \int_{\omega \mathbf{V}}^{\infty} \mathrm{E}_{\mathrm{g}}(\omega) \mathrm{d} \omega \sim \frac{\beta^{\prime 2}}{4} \sum_{\mathbf{R}} \times \\
& \times\left\langle Q^{\mathrm{R}^{2}} \int_{-\infty}^{+\infty} \int_{\omega \mathrm{V}}^{\infty} \exp \mathrm{j} \omega t\left(\Gamma_{x x}\left(\Omega^{\mathrm{R}}(t)\right) \Gamma_{y y}\left(\Omega^{\mathrm{R}}(0)\right) \mathrm{d} \omega \mathrm{d} t\right\rangle .\right.
\end{aligned}
$$

En intégrant d'abord sur les fréquences, puis sur les temps, on trouve sans difficultés :

$$
\begin{aligned}
& \int_{\omega \mathbf{V}}^{\infty} \mathrm{E}_{\mathbf{g}}(\omega) \mathrm{d} \omega \sim \frac{1}{2} \frac{\beta^{\prime 2}}{4} \sum_{\mathbf{R}} \times \\
& \times\left\langle Q^{\mathrm{R}^{2}} \Gamma_{x x}\left(\Omega^{\mathrm{R}}(0)\right) \Gamma_{y y}\left(\Omega^{\mathrm{R}}(0)\right)\right\rangle \\
& 72^{*}
\end{aligned}
$$


et de même

$$
\int_{\omega_{\mathbf{V}}}^{\infty} \mathrm{F}_{2 \mathrm{~g}}(\omega) \mathrm{d} \omega \sim \frac{1}{2} \beta^{\prime 2} \sum_{\mathbf{R}}\left\langle Q^{\mathrm{R}^{2}}\left(\Gamma_{x y}\left(\Omega^{\mathrm{R}}(0)\right)\right)^{2}\right\rangle,
$$

résultats qui dépendent de l'orientation instantanée des ions cyanures.

Le calcul se poursuit aisément par l'utilisation des harmoniques sphériques :

$$
\begin{aligned}
\int_{\omega \mathrm{V}}^{\infty} \mathrm{E}_{\mathrm{g}}(\omega) \mathrm{d} \omega & \sim \frac{4 \pi}{10} \beta^{\prime 2} \sum_{\mathrm{R}}\left\langle Q^{\mathrm{R}^{2}}\left(Y_{2}^{0}\left(\Omega^{\mathrm{R}}(0)\right)\right)^{2}\right\rangle \\
\int_{\omega_{\mathrm{V}}}^{\infty} \mathrm{F}_{2 \mathrm{~g}}(\omega) \mathrm{d} \omega & \sim \frac{6 \pi}{5} \beta^{\prime 2} \sum_{\mathrm{R}} \times \\
\times & \left\langle Q^{\mathrm{R}^{2}}\left(Y_{2}^{-1}\left(\Omega^{\mathrm{R}}(0)\right) Y_{2}^{1}\left(\Omega^{\mathrm{R}}(0)\right)\right)\right\rangle .
\end{aligned}
$$

Utilisons maintenant la fonction de probabilité d'orientation de trouver un ion cyanure dans la direction $\Omega$ à un instant donné. On développe cette fonction sur une base d'harmoniques cubiques compatibles avec la symétrie du problème ; ces harmoniques étant elles-mêmes développables (par construction) sur une base d'harmoniques sphériques, on a alors :

$$
\begin{aligned}
P(\theta, \varphi) & =\lambda_{0} Y_{0}^{0}(\theta, \varphi)+\lambda_{4} \times \\
\times & {\left[Y_{4}^{0}(\theta, \varphi)+\alpha\left(Y_{4}^{4}(\theta, \varphi)+Y_{4}^{-4}(\theta, \varphi)\right)\right]+\cdots }
\end{aligned}
$$

où $\alpha$ est une constante numérique définie par la symétrie cubique de $P(\theta, \varphi)$. Le calcul de valeur moyenne sur les orientations se résume donc à un calcul d'intégrales de la forme :

$$
\int Y_{l_{1}}^{m_{1}} Y_{l_{2}}^{m_{2}} Y_{l_{3}}^{m_{3}} \mathrm{~d} \Omega
$$

dont les valeurs sont aisément obtenues grâce aux coefficients de Wigner, dont les expressions sont tabulées pour les faibles valeurs de l'un des $l$ [26]. Les harmoniques sphériques intervenant dans les fonctions de corrélations de la diffusion Raman étant $Y_{2}^{-1}$, $Y_{2}^{1}$ et $Y_{2}^{0}$, on montre aisément que les seules harmoniques sphériques de $P(\theta, \varphi)$ pouvant donner une contribution non nulle sont dans ce cas précis $Y_{0}^{0}$ et $Y_{4}^{0}$.

Le coefficient $\lambda_{0}$ est déterminé par normalisation de la fonction $P(\theta, \varphi)$. Les expériences de diffusion Raman ne permettent donc la détermination que du coefficient $\lambda_{4}$, mais par opposition aux mesures de diffraction de neutrons, celui-ci est déterminé par une relation biunivoque, ce qui, compte tenu des incertitudes expérimentales des deux méthodes est un résultat fort intéressant.

Un calcul aisé montre que l'on a :

$$
\rho=3 \times \frac{1-\frac{4}{7} \frac{\lambda_{4}}{\lambda_{0}}}{1+\frac{6}{7} \frac{\lambda_{4}}{\lambda_{0}}}
$$

avec

$$
\lambda_{0}=\frac{1}{2 \sqrt{\pi}} \text { et } \rho=\frac{\int F_{2 g}(\omega) \mathrm{d} \omega}{\int \mathrm{E}_{\mathrm{g}}(\omega) \mathrm{d} \omega}
$$

soit

$$
\lambda_{4}=\lambda_{0} \times \frac{7}{6} \times \frac{3-\rho}{2+\rho} .
$$

Comme $0 \leqslant \rho \leqslant \infty$, le domaine de variation de $\lambda_{4} / \lambda_{0}$ est :

$$
-\frac{7}{6} \leqslant \frac{\lambda_{4}}{\lambda_{0}} \leqslant \frac{7}{4}
$$

Remarque. - Les bornes de ce domaine peuvent être atteintes et correspondent, comme on le vérifie, à des orientations cristallographiques simples des ions; on trouve en effet :

ions localisés sur les directions

$$
\begin{aligned}
& (1,0,0) \frac{\lambda_{4}}{\lambda_{0}}=\frac{7}{4} \\
& (1,1,1) \frac{\lambda_{4}}{\lambda_{0}}=-\frac{7}{6} \\
& (1,1,0) \frac{\lambda_{4}}{\lambda_{0}}=-\frac{7}{16} .
\end{aligned}
$$

L'isotropie implique $\lambda_{4}=0$, soit : $\rho=3$. Ce dernier résultat est en accord avec celui obtenu pour la mesure de la dépolarisation des raies de diffusion Raman en milieu isotrope [27]; avec les notations précédentes, on a en effet :

$$
I_{\mathrm{VV}}=\mathrm{A}_{1 \mathrm{~g}}+4 \mathrm{E}_{\mathrm{g}} \equiv 4 \mathrm{E}_{\mathrm{g}},
$$

dans notre cas, et

$$
I_{\mathrm{VH}}=\mathrm{F}_{2 \mathrm{~g}}
$$

soit en définitive

$$
\frac{I_{\mathrm{vH}}}{I_{\mathrm{VV}}}=\frac{3}{4}
$$

5.5 APPlication AUX SPECTRES DE BASSE FRÉQUENCE. ESTIMATEUR DE CORRÉLATION. - Le calcul précédent n'est valable qu'en haute fréquence, puisque c'est seulement dans ce cas que les spectres donnent des informations sur la fonction d'autocorrélation d'orientation des ions. Il est cependant possible de calculer $a$ priori le même rapport $\rho_{\mathrm{BF}}$ pour les spectres de basse fréquence. On voit aisément que ce rapport est indépendant du couplage dynamique et ne dépend que de l'ensemble des corrélations statiques entre les orientations moléculaires. L'égalité des rapports signifie simplement que les corrélations intermoléculaires n'affectent pas la mesure. 
L'interprétation de ce rapport, lorsqu'il diffère de sa valeur de haute fréquence, est plus délicate et nécessite des hypothèses supplémentaires. Dans celle où les corrélations d'orientation n'existent qu'entre plus proches voisins, on montre (voir annexe II) que le rapport $\rho_{\mathrm{BF}}$ mesuré dépend de $\lambda_{4}$ mais aussi de deux autres coefficients $\eta$ et $\eta^{\prime}$ définissant l'intensité de ces corrélations à courte distance. On en déduit ainsi :

$$
\rho_{\mathrm{BF}}=\frac{3\left(1-\frac{4}{7} \frac{\lambda_{4}}{\lambda_{0}}\right)+\eta}{1+\frac{6}{7} \frac{\lambda_{4}}{\lambda_{0}}+\eta^{\prime}}
$$

que l'on peut identifier formellement à :

$$
\rho_{\mathrm{BF}}=\frac{3\left(1-\frac{4}{7} \frac{\lambda_{4}^{\mathrm{c}}}{\lambda_{0}}\right)}{1+\frac{6}{7} \frac{\lambda_{4}^{\mathrm{c}}}{\lambda_{0}}} .
$$

On peut en déduire une relation reliant ces différents paramètres :

$$
\frac{\lambda_{4}^{\mathrm{c}}}{\lambda_{0}}=\frac{\lambda_{4}}{\lambda_{0}} \times\left(\frac{1}{1+\frac{\eta+2 \eta^{\prime}}{5}}+\frac{7}{30} \times \frac{3 \eta^{\prime}-\eta}{1+\frac{\eta+2 \eta^{\prime}}{5}}\right)
$$

avec, dans le cas d'un liquide : $3 \eta^{\prime}=\eta$ et $\lambda_{4}=0$.

Mais on constate que les résultats expérimentaux ne permettent pas de calculer chacun des coefficients, même dans le cas de l'hypothèse simple de corrélation entre plus proches voisins.

\subsection{APPLICATIONS : MESURES ET INTERPRÉTATION} POUR KCN ET NaCN. - La détermination du coefficient $\lambda_{4}^{c}$ est faite à partir des spectres de basses fréquences qui ont été corrigés de tous les phénomènes parasites, comme il a été dit dans la partie 3.4. Il est donc possible d'évaluer ce coefficient sur un grand intervalle de température. La mesure du coefficient $\lambda_{4}$ se fait à partir des spectres de haute fréquence (bandes latérales de la raie d'élongation), mais est difficile car le spectre de type $A_{1 g}$ est de forte intensité et de largeur non nulle, ce qui produit une pollution des spectres de type $E_{g}$ et $F_{2 g}$.

Une autre difficulté dans l'étude des spectres de basse fréquence provient du fait que le spectre en dessous de $10 \mathrm{~cm}^{-1}$ est difficilement mesurable par suite des défauts de polarisation et de la diffusion parasite qui peut apparaître. On conçoit que la précision sur la détermination du coefficient peut être médiocre. Les résultats que nous donnons ici ne sont donc qu'indicatifs, des résultats plus précis nécessitant la détermination précise du spectre de très basse fréquence selon, par exemple, une technique développée par [27].
$\mathrm{NaCN}$ : Les mesures expérimentales du coefficient $\lambda_{4}^{c}$ ont été reportées sur la figure 10 . On remarque un bon accord entre la valeur de $\lambda_{4}^{\mathrm{c}}$ mesurée en basse fréquence et la valeur mesurée par J. M. Rowe et al. $[3,4]$. Le domaine de température étudié est de $300 \mathrm{~K}$ à $450 \mathrm{~K}$. Dans cette étude, nous avons été limités d'une part par les possibilités de notre instrumentation, d'autre part, par l'apparition après un long chauffage sous vide, d'un dépoli des faces du cristal.

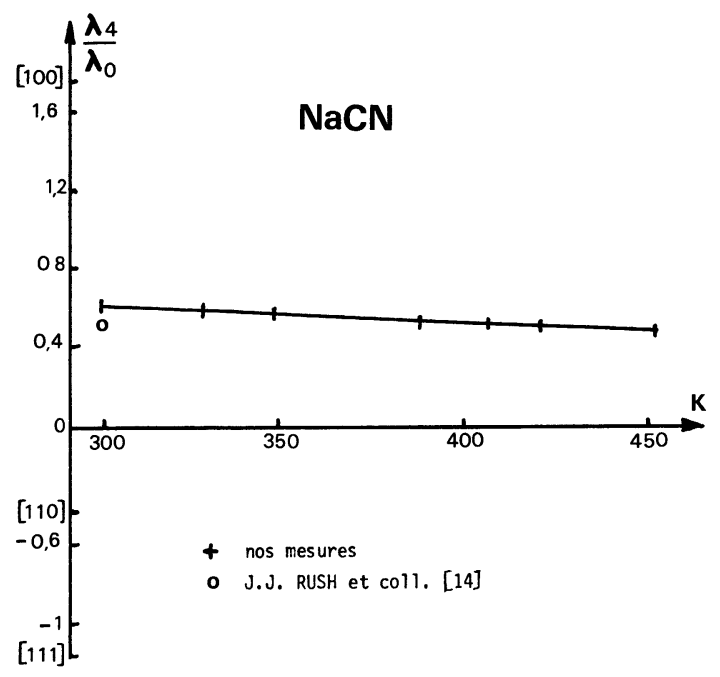

Fig. 10. - Evolution du rapport $\lambda_{4}^{\mathrm{c}} / \lambda_{0}$ en fonction de la température pour $\mathrm{NaCN}$.

$\left[\lambda_{4}^{\mathrm{c}} / \lambda_{0}\right.$ ratio versus temperature in sodium cyanide. $]$

A température ordinaire, l'accord entre nos résultats et celui de J. M. Rowe et al. [4] peut être interprété de deux manières différentes :

- les corrélations d'orientation entre les ions cyanures sont faibles ou non mesurables par cette technique,

- ce résultat n'est qu'une coïncidence et l'existence d'un spectre de diffusion de très basse fréquence modifierait ce résultat.

Nous pensons que cette dernière interprétation est plus raisonnable puisque nous montrerons que la durée de vie d'orientation $T_{\mathrm{r}}$ des ions cyanures dans une direction $(0,0,1)$ est au moins de l'ordre de $0,3 \mathrm{ps}$, ce qui correspond à un spectre de largeur inférieure à $15 \mathrm{~cm}^{-1}$ qui, dans nos conditions expérimentales, est impossible à déterminer.

Un tel spectre, comme nous le verrons (cf. 6.2) affecterait essentiellement le spectre de type $\mathrm{E}_{\mathrm{g}}$. On en déduit que le coefficient est certainement surestimé, donc que le coefficient $\lambda_{4}^{c}$ l'est aussi. L'évolution avec la température est faible mais conforme à l'idée que l'ordre à longue distance, diminue quand la température augmente, puisque $\lambda_{4}^{\mathrm{c}}$ est proportionnel à $\lambda_{4}$ et que ce dernier caractérise l'anisotrope de $P(\theta, \varphi)$. 
KCN : Les premiers résultats obtenus furent ceux

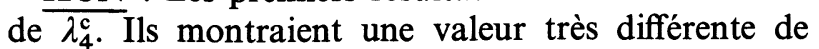
ceux obtenus par J. M. Rowe et al. [4].

Une discussion précise nous a montré que la différence entre ces deux résultats ne pouvait provenir d'une incertitude des expériences. Ce résultat suggérait donc l'existence de fortes corrélations dans ce cristal. Encore fallait-il vérifier que le coefficient $\lambda_{4}$ obtenu dans nos expériences était bien compatible avec celui trouvé par diffraction neutronique. Cette vérification étant très délicate, comme nous l'avons déjà dit, l'expérience n'a été réalisée qu'à $300 \mathrm{~K}$ qui est la température à laquelle J. M. Rowe et al. [4] avaient travaillé. Comme le montre la figure 11, l'accord est satisfaisant et il confirme le modèle proposé pour la diffusion Raman et l'écart entre $\lambda_{4}^{c}$ et $\lambda_{4}$ met bien en évidence l'existence de corrélation dans le cyanure de potassium.

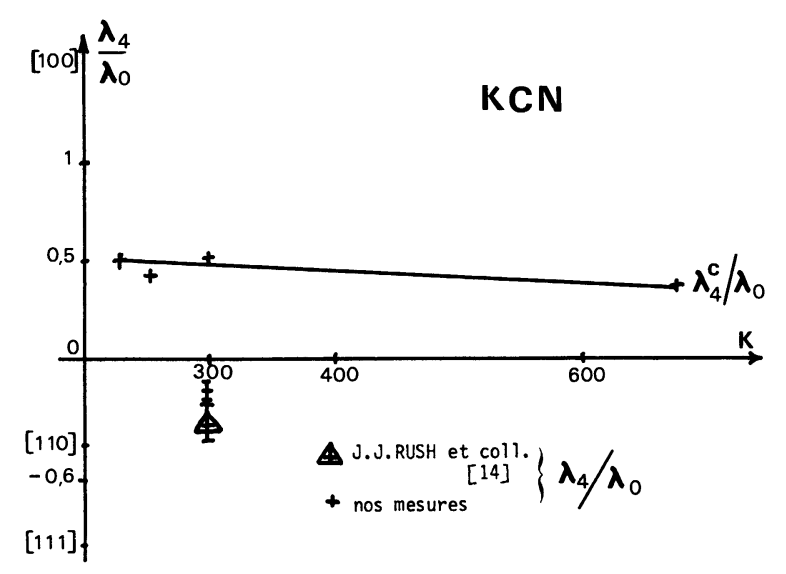

Fig. 11. - Evolution du rapport $\lambda_{4}^{c} / \lambda_{0}$ en fonction de la température pour $\mathrm{KCN}$.

$\left[\lambda_{4}^{c} / \lambda_{0}\right.$ ration versus temperature in potassium cyanide. $]$

Les résultats publiés, ici et précédemment [13], sont les premiers relatifs à la mesure de ce coefficient par des méthodes optiques. Nous avons pu retrouver la valeur du coefficient non corrélé $\lambda_{4}$, déterminer la valeur de $\lambda_{4}^{c}$, et montrer l'existence de corrélation dans le cas de cyanure de potassium.

Le cas du cyanure de sodium est moins clair puisque nous n'avons pu montrer l'existence de tels couplages. Cependant, nous devons souligner la difficulté expérimentale dans la détermination de ces coefficients, les spectres de haute fréquence étant d'intensité faible.

6. Spectres de basse fréquence de $\mathrm{NaCN}$. - Il est instructif de commencer l'étude des spectres de basse fréquence par la recherche des modes de libration puisque, en général, ces modes sont actifs en diffusion Raman. Nous allons montrer qu'il existe des règles de sélection qui sont satisfaites pour le cyanure de sodium, même dans ce cas de cristal plastique; nous étudierons ensuite l'existence de modes liés aux mouvements de réorientations et terminerons cette partie par une discussion sur les mouvements de libration.

6.1 RECHERCHE DES MODES DE LIBRATION. - Les cyanures étant désordonnés dans la phase I, il n'est pas possible d'appliquer la méthode de dénombrement habituelle. Cependant, l'utilisation de la méthode de Kastler-Rousset [28] permet dans certains cas favorables de déterminer l'activité des modes de libration.

Pour que l'on puisse utiliser ce procédé, il est nécessaire que les librations n'introduisent pas de modification du tenseur de polarisabilité de l'ion, hypothèse que nous avons vérifiée dans la partie 4.3. En étudiant l'existence de libration autour d'une direction particulière, on montre que les librations ne peuvent pas apparaître dans les spectres de type $E_{g}$, si elles s'effectuent autour de direction $(1,0,0)$. Dans tous les autres cas (librations autour de directions $(1,1,1)$ $(1,1,0))$, les modes de librations apparaissent aussi bien dans les deux spectres de symétrie $E_{g}$ et $F_{2 g}$, ce résultat étant valable quelles que soient les corrélations entre les mouvements des ions et leurs orientations. Nous avons ainsi un renseignement important sur l'orientation des ions cyanures dans le cyanure de sodium car dans le spectre de type $\mathrm{E}_{\mathbf{g}}$ (Fig. 5), on observe seulement un pic central. Celui-ci ne peut être attribué au mode de libration, puisque ce pic existe encore dans le spectre non polarisé de la phase II où les deux modes de librations sont mesurables. D'autre part, on conçoit mal l'existence d'un mode de libration qui serait suramorti dans le spectre de type $E_{g}$ et non suramorti dans le spectre de type $F_{2 g}$. Il est donc vraisemblable que dans le cyanure de sodium, les ions $\mathrm{CN}^{-}$librent autour de directions $(1,0,0)$.

Dans le cas du cyanure de potassium, le mode de libration apparaît cette fois, aussi bien dans le spectre de type $E_{g}$ que dans celui de type $F_{2 g}$ (Fig. 6). Les librations ne se font donc certainement pas uniquement autour de la direction $(1,0,0)$.

Les expériences de diffusion Raman ne permettent pas actuellement de préciser la direction, ou les directions, probables. Notons que nous ne pouvons exclure une situation plus complexe où les ions cyanures pourraient librer autour de direction $(1,0,0)$ d'une part, et d'autre part, autour d'autres directions permises par la symétrie du cristal.

6.2 INTERPRÉTATION DES SPECTRES DE TYPE $\mathrm{E}_{\mathrm{g}}$ DU CYANURE DE SODIUM. - Dans ce cas, le spectre de diffusion Raman n'est dû qu'aux mouvements de réorientation des ions, à partir de directions $(1,0,0)$. Rappelons que la valeur de l'intensité diffusée dans ce type de symétrie est :

$$
\mathrm{E}_{\mathrm{g}} \sim \frac{\beta^{2}}{4} \text { T.F. } \sum_{\mathbf{R}, \mathbf{R}^{\prime}}\left\langle\Gamma_{i i}\left(\Omega^{\mathrm{R}}(t)\right) \Gamma_{j j}\left(\Omega^{\mathrm{R}^{\prime}}(0)\right)\right\rangle
$$


ce que l'on peut encore écrire, si $\theta$ est l'angle déterminant la position de l'ion cyanure $\mathrm{R}$ à l'instant $t$

$$
\begin{aligned}
\mathrm{E}_{\mathbf{g}} \sim \frac{\beta^{2}}{4} \mathrm{~T} . \mathrm{F} \cdot \sum_{\mathbf{R}, \mathbf{R}^{\prime}}\left\langle\left(3 \cos ^{2} \theta^{\mathrm{R}}(t)-1\right) \times\right. & \\
& \left.\times\left(3 \cos ^{2} \theta^{\mathbf{R}^{\prime}}(0)-1\right)\right\rangle .
\end{aligned}
$$

Cette fonction de corrélation d'orientation dépend explicitement de $T_{\mathrm{r}}$ - durée moyenne de libration et de $T_{\mathrm{R}}$ - durée moyenne de réorientation. Dans notre modèle, nous affirmons que la réorientation n'est possible que durant le temps où, par suite du mouvement relatif des ions voisins, les forces de répulsion sont suffisamment faibles. Durant ce temps, nous pourrons donc considérer que la rotation des ions est quasi libre. On suppose, en outre, que cette rotation se situe dans un plan puisqu'il semble peu probable que les déplacements soient tels que tous les plus proches voisins se déplacent en même temps et d'une manière synchrone.

En négligeant, en première approximation, les corrélations dynamiques entre les ions cyanures, l'intensité diffusée est proportionnelle à :

$$
\mathrm{E}_{\mathrm{g}} \sim \frac{\beta^{2}}{4} \mathrm{~T} . \mathrm{F} .\left\langle 3 \cos ^{2}(\Omega t)-1\right\rangle,
$$

où $\Omega$ est la vitesse angulaire de rotation de l'ion cyanure.

La rotation ne se faisant qu'entre deux directions voisines $(1,0,0)$, on en déduit :

$\mathrm{E}_{\mathrm{g}} \sim \beta^{2} \int_{0}^{\infty} p(\Omega) \mathrm{d} \Omega \int_{0}^{\pi / 2 \Omega}\left(3 \cos ^{2} \Omega t-1\right) \times$

$$
\times \cos \omega t \mathrm{~d} t .
$$

Comme la fonction de densité de probabilité d'avoir la vitesse angulaire $\Omega$ dans le plan de rotation est :

$$
p(\Omega)=\exp -\frac{I \Omega^{2}}{2 k T}
$$

on trouve

$$
\mathrm{E}_{\mathrm{g}}(\omega) \sim \beta^{2} \int_{0}^{\infty} \frac{4 \Omega^{2}+2 \omega^{2}}{\omega\left(4 \Omega^{2}-\omega^{2}\right)} \sin \frac{\omega \pi}{2 \Omega} \exp -\frac{\Omega^{2}}{\Omega_{0}^{2}} \mathrm{~d} \Omega
$$

avec

$$
\Omega_{0}=\sqrt{\frac{2 k T}{I}}
$$

ce que l'on peut encore écrire :

$$
\mathrm{E}_{\mathrm{g}}(x) \sim \frac{\beta^{2}}{2} \int_{0}^{\infty} \frac{1}{u^{2}} \times \frac{1+2 u^{2}}{1-u^{2}} \sin (\pi u) \exp -\frac{x^{2}}{u^{2}} \mathrm{~d} u
$$

avec

$$
x=\frac{\omega}{2 \Omega_{0}} .
$$

Cette intégrale converge pour toutes valeurs de $x$ différentes de zéro. $\mathrm{E}_{\mathrm{g}}(\omega)$ est donc obtenu à une constante de normalisation près que l'on détermine par intégration. On constate sur la figure 12 que le spectre calculé pour $T=300 \mathrm{~K}$ s'enroule autour du spectre expérimental, ce qui traduit un très bon accord entre notre modèle et les résultats expérimentaux. Un aussi bon accord a été retrouvé pour toutes températures étudiées $(300 \mathrm{~K} \leqslant T \leqslant 450 \mathrm{~K})$, alors que la largeur à mi-hauteur du spectre varie de $33 \mathrm{~cm}^{-1}$ à $40 \mathrm{~cm}^{-1}$.

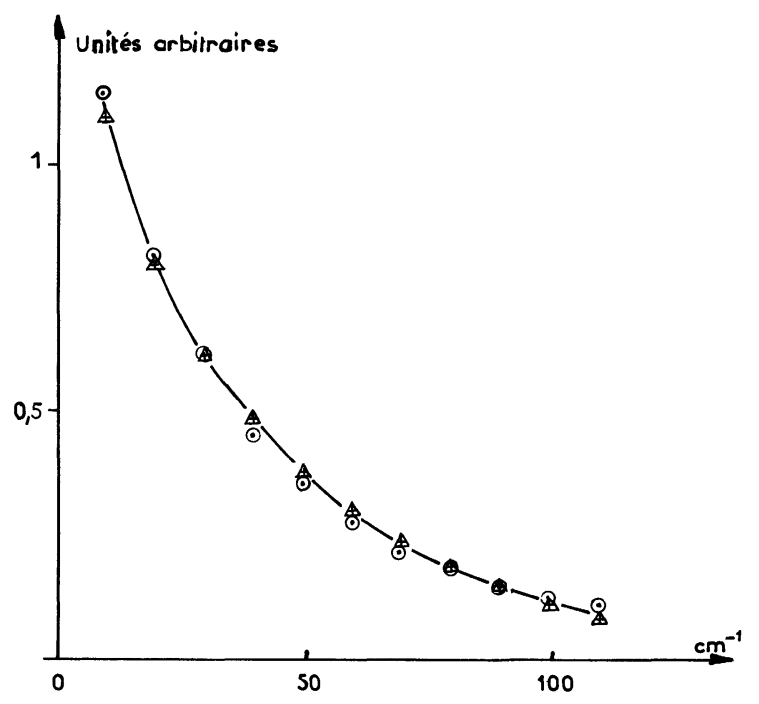

Fig. 12. - Spectre de type $\mathrm{E}_{\mathrm{g}}$ de $\mathrm{NaCN}(T=300 \mathrm{~K})$. $\triangle$ Valeurs expérimentales; $\odot$ Valeurs calculées d'après la formule (20).

$\left[\mathrm{E}_{\mathrm{g}}\right.$ type spectrum of $\mathrm{NaCN}(\mathrm{T}=300 \mathrm{~K}) . \mathbb{A}$ Experimental values; $\odot$ Values calculated from (20) formula.]

Ceci confirme la validité des hypothèses faites : la réorientation est bien effectuée entre deux directions $(1,0,0)$ voisines et cette réorientation est bien quasi libre. D'autre part, il semble raisonnable que cette orientation s'effectue dans un plan $(1,0,0)$. Par ailleurs, on déduit de ce modèle un temps moyen de réorientation :

$$
T_{\mathrm{R}}=\int_{0}^{\infty} p(\Omega) \times \frac{\pi}{2 \Omega} \mathrm{d} \Omega=\frac{\pi}{2} \sqrt{\frac{I}{k T}}
$$

soit par exemple, pour $T=300 \mathrm{~K}, T_{\mathrm{R}}=0,30$ ps et pour $T=453 \mathrm{~K}, T_{\mathrm{R}}=0,24$ ps.

Dans le calcul précédent, nous n'avons pas fait intervenir le temps moyen de libration des ions cyanures dans leur site et le bon accord entre spectres calculés et mesurés montre que ce temps n'a pas d'influence pratique sur la forme du spectre, donc qu'il est certainement très supérieur au temps $T_{\mathrm{R}}$ déterminé, soit $T_{\mathrm{r}} \gg 0,3 \mathrm{ps}$. On peut cependant définir un ordre de grandeur du majorant de $T_{\mathrm{r}}$, en remarquant que les expériences de diffusion Brillouin et que la propagation des ondes ultrasonores appartiennent au même régime de propagation (cf. 3 ) et que la transition entre 
les deux régimes se fait, pour $\mathrm{NaCN}$, pour $\sigma=2 \mathrm{~cm}^{-1}$; soit $T_{\mathrm{r}}<2,5 \mathrm{ps}$. C'est donc dans le domaine des très basses fréquences Raman que devrait être recherché l'effet de la durée de vie moyenne des librations $\left(2 \mathrm{~cm}^{-1}<\sigma<18 \mathrm{~cm}^{-1}\right)$.

Remarque I. - Le spectre théorique est obtenu sans autre constante ajustable que la constante de normalisation correspondant à l'efficacité de la diffusion. Il peut aussi être ajusté sur un spectre d'allure lorentzienne, avec une assez bonne précision. Cependant, dans ce cas, la valeur de la largeur à mi-hauteur est donnée par l'expérience. Cette forme, analytiquement plus simple, sera utilisée ultérieurement dans le cas du cyanure de potassium.

Remarque II. - L'étude de l'évolution de la largeur $\Gamma$ du pic central en fonction de la température représente l'argument le plus fort contre un modèle de réorientation par passage par-dessus une barrière fixe de potentiel. En exprimant cette largeur sous la forme $\Gamma=\Gamma_{0} \exp (-E / k T)$, ce qui est possible et donne un bon accord avec les résultats expérimentaux, on trouve que cette barrière $E$ serait égale à $0,320 \mathrm{~kJ}$ soit $\sim 160 \mathrm{~K}$, ce qui est inférieur à la température du cristal et suggérerait que les ions sont en rotation libre, ce qui est en contradiction avec les données expérimentales de diffraction de neutrons.

6. 3 SPECTRES DE Diffusion DE TYPE $\mathrm{F}_{2 \mathrm{~g}}$ DE NaCN. - Comme nous l'avons dit précédemment, les librations peuvent apparaître dans les spectres de type $F_{2 g}$. En outre, les modes de réorientation peuvent aussi apparaître en basse fréquence, comme dans les spectres de symétrie $\mathrm{E}_{\mathrm{g}}$. Expérimentalement, le spectre de type $F_{2 \mathrm{~g}}$ apparaît comme une très large bande (voir figure 5) sans pic central très prononcé. Si la densité d'état des modes de libration pouvait être considérée comme un mode unique élargi par l'anharmonicité et de forme lorentzienne, on aurait :

$$
S(\omega) \sim(\bar{n}(\omega)+1) \times \frac{\Gamma \omega}{\left(\omega^{2}-\omega_{0}^{2}\right)^{2}+4 \Gamma^{2} \omega^{2}} .
$$

Il est certain que le cas étudié ici est plus compliqué ; cependant, afin de permettre une discussion numérique, nous allons appliquer au spectre expérimental une transformation ayant pour but de tenir compte de l'effet de température introduit par le facteur de BoseEinstein et de diminuer l'importance des spectres de basse fréquence. Cette transformation est la suivante :

$$
S^{\prime}(\omega)=S(\omega) \times \omega /(\bar{n}(\omega)+1)
$$

où $S(\omega)$ est le spectre mesuré expérimentalement; dans l'hypothèse d'une forme lorentzienne, le maximum de cette fonction $S^{\prime}$ coïncide avec $\omega_{0}$. La figure 13 montre l'évolution de la fonction $S^{\prime}(\omega)$ avec la température, tandis que la figure 14 montre l'évolution de la fréquence $\omega_{0}$ du maximum de $S^{\prime}$.

A partir de la transformation numérique précé-

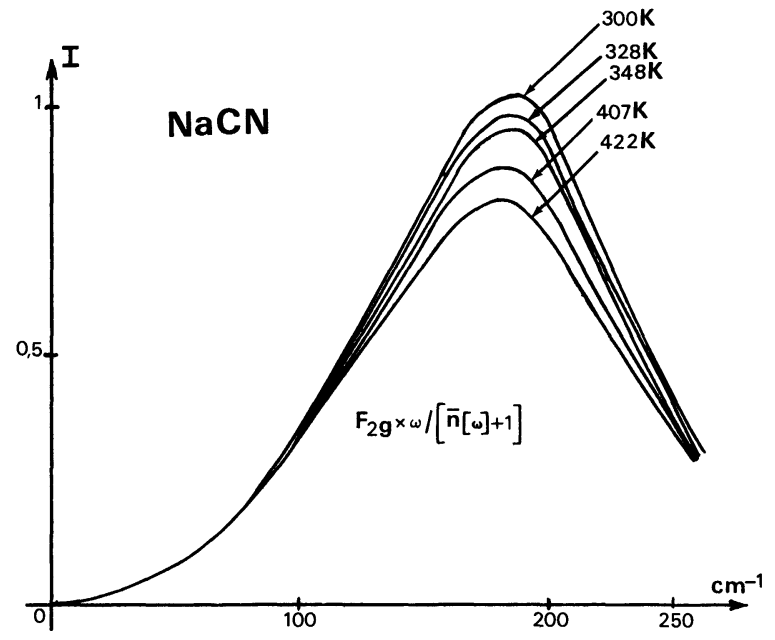

Fig. 13. - Evolution avec la température des spectres $F_{2 g}$ après transformation. Les différents spectres obtenus sont dans la même échelle d'intensité.

[Variation of $\mathrm{F}_{2 \mathrm{~g}}$ spectra with temperature after transformation. The same intensity scale is used for all spectra.]

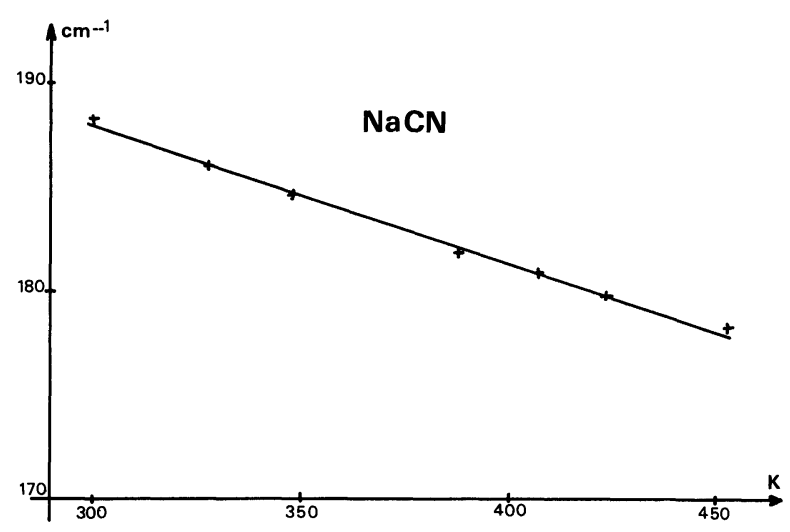

Fig. 14. - Evolution de la fréquence de libration avec la température.

[Variation of the librational frequency with temperature.]

dente, nous avons déterminé la largeur de cette bande de libration. On trouve, pour la largeur à mi-hauteur, une valeur $\Gamma=90 \mathrm{~cm}^{-1} \pm 2 \mathrm{~cm}^{-1}$ pratiquement indépendante de la température entre $300 \mathrm{~K}$ et $543 \mathrm{~K}$. D'autre part, l'évolution de la fréquence $\omega_{0}$ est aussi très faible avec la température, puisque l'on a (Fig. 14)

$$
\gamma=-\frac{1}{\omega_{0}} \frac{\mathrm{d} \omega_{0}}{\mathrm{~d} T}=3,6 \times 10^{-4} \mathrm{~K}^{-1} .
$$

La largeur du mode de libration a une origine complexe dont on peut au moins énumérer les diverses contributions :

- la libration a une durée de vie finie,

- le désordre lui-même joue un double rôle, puisqu'il intervient au niveau de la dynamique du cristal et de la détection Raman.

Nous avons montré que la largeur associée à la durée 
de vie des librations était comprise entre $2 \mathrm{~cm}^{-1}$ et $15 \mathrm{~cm}^{-1}$; par conséquent, la durée de vie est pratiquement sans influence dans le processus d'élargissement de la raie de libration qui est de l'ordre de $90 \mathrm{~cm}^{-1}$. L'origine de cet élargissement est donc à rechercher dans le désordre d'orientation des ions cyanures. $\mathrm{Si}$ le cristal était ordonné, les ions sodium voisins créeraient une barrière de potentiel $V_{0}$ au fond de laquelle oscillerait l'ion cyanure. En première approximation, le mode de libration n'aurait qu'une faible dispersion, liée au couplage translation-libration, comme on la rencontre dans les cristaux parfaits. En fait, le désordre des ions cyanures voisins modifie profondément la courbure de ce potentiel, soit directement par des couplages entre les ions $\mathrm{CN}^{-}$, soit par l'intermédiaire du déplacement des ions sodium. Le très grand désordre d'orientation des ions cyanures implique une forte dispersion des modes de libration, cette dispersion pouvant être du même ordre de grandeur que $V_{0}$. Il n'est plus alors possible d'effectuer le calcul de perturbation classiquement utilisé dans les cristaux désordonnés. Ce cas est plus à rapprocher du cas des solides amorphes et relève donc directement de méthodes telles que la dynamique moléculaire.

L'analyse précédente permet de mieux cerner la signification physique de la fréquence de libration nommée précédemment $\omega_{0}$ : elle n'est en fait que le barycentre de ces fréquences. On peut ainsi comprendre l'évolution de celle-ci avec la température, en remarquant que, lorsque la température augmente, les distances entre les ions augmentent aussi, si bien que la barrière de potentiel moyenne diminue et avec elle, les fréquences de librations, ainsi que leur barycentre. L'évolution des largeurs est plus compliquée. La variation de cette largeur avec la température n'èst liée qu'aux fluctuations locales des couplages intermoléculaires; celle-ci peut ne diminuer que fort lentement lorsque la température augmente, ce qui peut expliquer, avec l'importance des erreurs expérimentales, que la largeur paraisse constante avec la température.

Notons enfin, d'après la figure 13 qui montre l'évolution thermique du spectre $\mathrm{F}_{2 \mathrm{~g}}$ corrigé du facteur de Bose-Einstein, que l'intensité de ce spectre diminue quand la température augmente. Ceci est normal car, plus cette dernière croît, plus le nombre d'ions en réorientation augmente et par conséquent plus le nombre d'ions en libration diminue. L'intensité du spectre $\mathrm{F}_{2 \mathrm{~g}}$ étant proportionnelle au nombre de ces derniers diminue donc aussi.

7. Essai d'interprétation des spectres de basse fréquence de KCN. - Nous allons essayer d'interprétër les résultats expérimentaux obtenus pour le cyanure de potassium à l'aide des modèles utilisés pour le cyanure de sodium. Nous rencontrerons ici des difficultés provenant de ce que les librations ne se font pas uniquement autour de directions $(1,0,0)$ de la maille cubique (cf. 7.1). Ceci est en accord avec les résultats de J. M. Rowe et al. [4] (que nous avons rappelés dans la première partie) qui trouvent que la fonction de probabilité présente un maximum principal dans la direction $(1,1,1)$.

7.1 Modes DE RÉORIENTATION. - D'après la forme de la fonction de probabilité des ions cyanures, les modes associés aux réorientations peuvent apparaître aussi bien dans les spectres de type $\mathrm{E}_{\mathrm{g}}$ que de type $F_{2 g}$. Ces modes apparaissent sous forme d'un pic d'allure lorentzienne (cf. 7, remarque 1). L'inspection des spectres expérimentaux montre que ce pic semble absent en $F_{2 g}$ (ou du moins de très faible intensité par rapport aux modes de librations). En $\mathrm{E}_{\mathrm{g}}$, ce spectre apparaît et il est d'une intensité comparable à celle des modes de librations. Seul ce dernier spectre est pratiquement exploitable numériquement pour l'étude des modes de réorientation. Afin de déterminer un ordre de grandeur du temps moyen de réorientation $\left(T_{\mathrm{R}}\right)$, nous avons admis que ce spectre était la somme d'un spectre de libration, de forme inconnue et d'un processus de réorientation analogue à celui existant dans $\mathrm{NaCN}$. Ce dernier sera décrit par une lorentzienne de largeur à ajuster, puisque nous avons montré dans le cas du cyanure de sodium que la forme de ce spectre pouvait être assez bien approximée par une lorentzienne et, d'après le modèle proposé, cette forme ne dépend pas de l'angle de réorientation. La décomposition du spectre montre que la largeur du mode de réorientation est pratiquement constante avec la température et de l'ordre de $30 \mathrm{~cm}^{-1}\left( \pm 2 \mathrm{~cm}^{-1}\right.$ entre $200 \mathrm{~K}$ et $450 \mathrm{~K}$ ). Cette largeur est du même ordre de grandeur que celle déterminée pour le cyanure de sodium (entre 33 et $40 \mathrm{~cm}^{-1}$ ) et montre que l'origine de ce pic central (ou aile Rayleigh large), est la même dans ces deux corps.

Ce résultat conduit à deux remarques :

- dans le modèle proposé (cf. 7.2) la forme du spectre ne dépend que de la valeur du moment d'inertie de l'ion, de la température et de l'angle de rotation (pris égal, dans le cas de $\mathrm{NaCN}$, à $\pi / 2$ ). Si le processus envisagé (rotation quasi libre entre deux directions $(1,0,0)$ de la maille cubique) était exactement le même dans les deux composés, les spectres ne devraient dépendre que de la température et donc être de même largeur à même température. La différence observée entre les deux résultats expérimentaux n'est pas en contradiction avec cette affirmation puisque la méthode utilisée dans le cas de $\mathrm{KCN}$ est assez grossière et ne permet pas l'obtention d'une précision suffisante. Cependant le rôle des différences structurelles devraient aussi être envisagées si l'on possédait une meilleure méthode de décomposition des spectres. La difficulté fondamentale est l'inexistence d'une théorie permettant de décrire simultanément la réorientation des molécules et leurs mouvements de librations; notre méthode de séparation ne peut donc donner qu'une description approchée des spectres et un ordre de grandeur des valeurs caractéristiques. 
7.2 EVOLUTION DU MODE DE LIBRATION AVEC LA TEMPÉRATURE. - Les spectres de librations sont obtenus soit directement (spectre $\mathrm{F}_{2 \mathrm{~g}}$ ), soit par soustraction dans le cas du spectre $\mathrm{E}_{\mathrm{g}}$ (cf. $\S$ précédent). Les figures 15 et 16 montrent l'évolution de ces spectres après qu'ils aient été transformés suivant la méthode décrite pour le cyanure de sodium (cf. formule (21)). Ces spectres évoluent fortement avec la température.

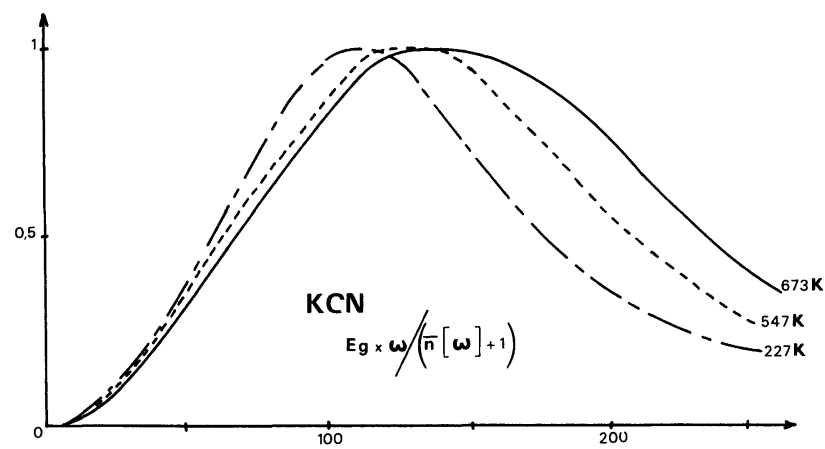

Fig. 15. - Evolution, avec la température, des spectres $S^{\prime}$ obtenus à partir des spectres de type $\mathrm{E}_{\mathrm{g}}$. Les spectres ont été renormalisés.

[Variation of the $S^{\prime}$ spectra, obtained from $E_{g}$ spectra, with temperature. The spectra are renormalized.]

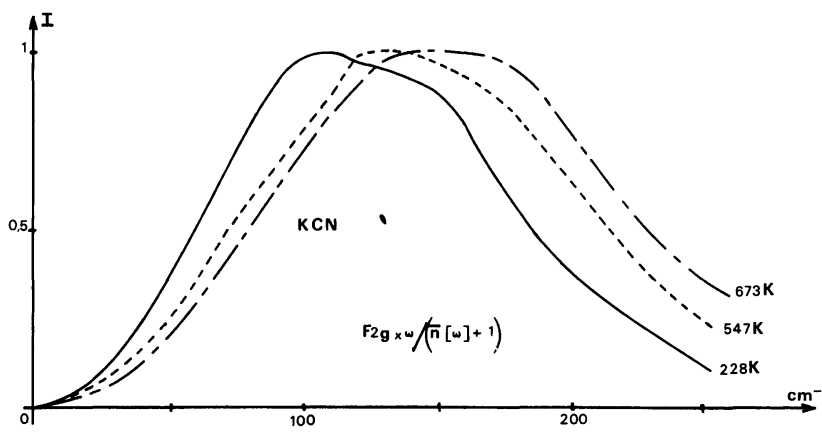

Fig. 16. - Evolution, avec la température, des spectres $S^{\prime}$ obtenus à partir des spectres de type $\mathrm{F}_{2 \mathrm{~g}}$. Les spectres ont été renormalisés.

[Variation of the $S^{\prime}$ spectra, obtained from $F_{2 g}$ spectra, with temperature. The spectra are renormalized.]

On constate, notamment, que la fréquence du maximum augmente quand la température croît, ce qui est le contraire de l'évolution constatée sur $\mathrm{NaCN}$. Ce comportement peut s'interpréter qualitativement en utilisant le même raisonnement que pour le cyanure de sodium. En effet, nous avons montré qu'il existe dans KCN de fortes corrélations des ions cyanures (notamment par comparaison des coefficients $\lambda_{4}$ et $\lambda_{4}^{c}$ ) et une évolution de celles-ci avec la température. La présence de corrélations d'orientations des ions dans le cristal, d'une part, pondère plus fortement le centre de la zone de Brillouin dans la densité d'état mesurée en diffusion Raman, et d'autre part, diminue les fluctuations du potentiel de libration. Si le centre de la zone de Brillouin est un minimum de la courbe de dispersion des librations (dans l'approximation des petites fluctuations du potentiel de libration), on s'attend, lorsque la température augmente, à ce que la fréquence moyenne soit plus élevée et la largeur plus grande, puisque la diminution des corrélations permet de voir toute la zone de Brillouin, et augmente les fluctuations de potentiel. C'est bien ce qui est expérimentalement trouvé

8. Discussion et conclusion. - L'étude et l'interprétation des spectres de diffusion Raman des cristaux plastiques présentent de nombreuses difficultés tant expérimentales que théoriques. Du point de vue expérimental, nous avons pu mettre au point une méthode de correction des spectres qui nous a permis d'obtenir des informations quantitatives sur les différents spectres $A_{1 g}, E_{g}$ et $F_{2 g}$. Ceci nous a permis de confirmer par une méthode optique l'anisotropie de la fonction de probabilité d'orientation des ions $\mathrm{CN}^{-}$dans les deux cyanures, anisotropie qui avait déjà été mesurée par diffraction de neutrons. Par ailleurs, notre effort a été de montrer que des idées relativement simples, telles que des mouvements réorientationnels d'ions, couplés aux mouvements de translations des ions voisins, permettent de rendre compte qualitativement des spectres observés.

Dans le cas du cyanure de sodium, nous avons mis en évidence l'existence d'une bande de libration, ce que les expériences de diffusion inélastique de neutrons n'avaient pu montrer. Notre réussite, à cet égard, tient à deux facteurs indépendants : l'un, fortuit, est que les règles de sélection de la diffusion Raman nous ont permis d'isoler cette bande, malgré sa largeur; l'autre est que, dans les cristaux étudiés ici, la diffusion Raman n'est due qu'aux seuls mouvements angulaires des ions $\mathrm{CN}^{-}$. A l'inverse, la diffusion neutronique est sensible à tous les mouvements, y compris ceux de translations : le couplage fort entre translations et rotations superposé à la largeur de la bande de libration et à la faible durée de vie de ces dernières explique, à notre avis, les échecs précédents.

Nous avons aussi montré que les phénomènes de réorientations des ions $\mathrm{CN}^{-}$pouvaient se décrire comme le piégeage des ions au fond de puits de potentiels pendant un temps $T_{\mathrm{r}}$, suivi directement d'une réorientation vers un autre puits, qui se fait en un temps $T_{\mathbf{R}}$ accessible dans nos expériences. En effet, le spectre de réorientation $\mathrm{E}_{\mathrm{g}}$ s'interprète complètement comme des orientations quasi libres planaires entre deux directions voisines $(1,0,0)$ avec un temps moyen de réorientation de l'ordre de 0,25 ps qui varie relativement lentement avec la température. D'autre part, nous avons pu donner un ordre de grandeur de la durée moyenne de libration qui doit être comprise entre :

$$
0,3 \mathrm{ps} \leqslant T_{\mathrm{r}} \leqslant 2,5 \mathrm{ps}
$$


Le cas du cyanure de potassium est plus compliqué puisque les directions de librations n'ont pu être précisées (mais ne sont pas uniquement des directions $(1,0,0))$. Nous avons pu montrer que les traits généraux trouvés pour le cyanure de sodium se retrouvaient dans ce composé; une différence existe bien dans le comportement du spectre de libration avec la température; cependant, l'adjonction du rôle des corrélations d'orientation, dans le modèle utilisé pour le cyanure de sodium, permet de rendre compte de cette évolution.

Le modèle proposé pour les processus de réorientations doit être comparé d'abord aux résultats fournis par R.M.N. [1, 2]. Celle-ci donne un temps moyen de libration d'un ion cyanure dans une direction donnée de $T_{\mathrm{r}}=120 \mathrm{ps}$ pour $\mathrm{NaCN}$ à $300 \mathrm{~K}$ et $\mathrm{T}_{\mathrm{r}}=230 \mathrm{ps}$ pour $\mathrm{KCN}$ à la même température. Ces durées sont environ 100 fois plus longues que celles que nous avons estimées. Nous pensons que l'interprétation des mesures de R.M.N. est à reconsidérer. En effet, un tel temps de vie est du même ordre de grandeur que celui trouvé dans l'adamantane [30], corps dans lequel les librations quoique très larges, ont pu être détectées par diffusion inélastique de neutrons; la différence de comportement entre ces deux corps serait assez difficile à comprendre. De plus, on devrait mettre en évidence un changement dans les courbes de dispersions de phonons aux environs de $0,25 \mathrm{~cm}^{-1}$, c'est-àdire une modification de la vitesse du son entre les expériences ultra-sonores et celles de diffusion Brillouin; ceci n'est pas le cas, alors que ce changement est visible à beaucoup plus haute fréquence (cf. partie 3).

D'un autre côté, les expériences de diffusions inélastiques de neutrons de J. J. Rush et al. [22] ont été récemment réanalysées par ces auteurs et $\mathrm{K}$. $\mathrm{H}$. Michel et al. [24]. Dans cette dernière publication, les auteurs mettent en évidence, dans KCN, l'existence d'un pic central, visible en dehors du centre de la zone de Brillouin, et dont la largeur est approximativement égale à celle trouvée par nous dans les deux cyanures. Leur analyse diffère cependant fortement de la nôtre, car ils attribuent ce pic aux fluctuations de basse fréquence de la probabilité d'orientation des molécules, et le spectre total proviendrait du couplage entre les fluctuations et les phonons acoustiques. On peut penser que ce mécanisme ne peut guère expliquer nos spectres $\mathrm{E}_{\mathrm{g}}$; en effet, c'est ce couplage qui est responsable de la transition de phase (phase I-phase II), si bien que le pic de fluctuation correspondant devrait être surtout visible dans les spectres $F_{2 \mathrm{~g}}$ [14]. Or, à basse fréquence, l'intensité du spectre $\mathrm{E}_{\mathrm{g}}$ est nettement plus grande que celle du spectre $F_{2 g}$ (Fig. 5).

Par ailleurs, comme nous l'avons déjà dit, le changement de la vitesse du son se fait, pour les phonons transverses se propageant avec la vitesse liée à $c_{44}$, pour une fréquence $v_{0}$ de l'ordre de $60 \mathrm{GHz}$ (cf. 2.2). Si ce changement de régime est dû au couplage des phonons acoustiques avec les fluctuations d'orientation [14], le temps de vie de ces derniers est approximativement égal à $1 / 2 \pi v_{0}$ soit 2,5 ps. Il paraît assez étonnant que ce temps soit d'un ordre de grandeur plus court dans la représentation $\mathrm{E}_{\mathrm{g}}$. Ce sont ces deux raisons qui nous ont conduits à proposer une interprétation différente pour ce spectre. Cependant, la grande similitude entre les largeurs mesurées par J. J. Rush et al. [4] reste surprenante et l'on peut se demander si le mécanisme que nous avons invoqué ne pourrait pas aussi expliquer les résultats de neutrons. Seule l'étude comparée des spectres dans différentes zones de Brillouin permettrait de faire progresser notre compréhension à cet égard.

\section{Annexe I}

Exemple de correction des spectres de diffusion Raman transverse. - Soit une onde incidente polarisée verticalement, par exemple. Au point où se produit la diffusion, le champ électrique peut se décomposer sur deux axes ( $z$ vertical, $x$ horizontal) :

$$
E_{z}=E_{0} \sqrt{1-\alpha} \mathrm{e}^{j \varphi} \mathrm{e}^{j \omega t}\left({ }^{2}\right), \quad E_{x}=E_{0} \sqrt{\alpha} \mathrm{e}^{j \omega t} .
$$

D'après Born, l'intensité diffusée, dont l'état de polarisation est parallèle à $z$ (ici vertical) est :

$$
I^{\mathrm{V}}=\left|E_{0}^{\mathrm{V}}\right|^{2}\left[(1-\alpha) I_{z z, z z}+\alpha I_{z x, z x}\right],
$$

celle dont l'état de polarisation est $x$ (ici horizontal) est :

$$
I^{\mathrm{H}}=\left|E_{0}^{\mathrm{V}}\right|^{2} I_{z x, z x} .
$$

Sur le trajet de sortie, l'état de polarisation de l'onde diffusée est modifié ; comme précédemment, on a :

$$
J^{\mathrm{V}}=(1-\beta) I^{\mathrm{V}}+\beta I^{\mathrm{H}}, \quad J^{\mathrm{H}}=\beta I^{\mathrm{H}}+(1-\beta) I^{\mathrm{V}} .
$$

$\left(^{2}\right)$ Les coefficients $\alpha$ (ou $\beta$ ) représentent le taux de dépolarisation de l'onde électromagnétique, l'angle $\varphi$ le déphasage entre l'onde polarisée verticalement et horizontalement. 
En résumé, on a :

$$
\begin{aligned}
& I^{\mathrm{VV}}=I_{0}^{\mathrm{V}}\left[(1-\alpha)(1-\beta) I_{x x, x x}+(\beta+(1-\beta) \alpha) I_{x y, x y}\right] \\
& I^{\mathrm{VH}}=I_{0}^{\mathrm{V}}\left[(1-\alpha) \beta I_{x x, x x}+(\alpha \beta+1-\beta) I_{x y, x y}\right] \\
& I^{\mathrm{HV}}=I_{0}^{\mathrm{H}}\left[\alpha(1-\beta) I_{x x, x x}+((1-\alpha)(1-\beta)+\beta) I_{x y, x y}\right] \\
& I^{\mathrm{HH}}=I_{0}^{\mathrm{H}}\left[(1-\beta)+\beta(1-\alpha) I_{x y, x y}+\alpha \beta I_{x x, x x}\right]
\end{aligned}
$$

où $I_{0}^{\mathrm{H}}$ et $I_{0}^{\mathrm{V}}$ sont respectivement les valeurs de l'intensité du rayon lumineux d'excitation de polarisation $\mathrm{V}$ ou $\mathrm{H}$.

Il est possible de mesurer directement les coefficients $\alpha$ et $\beta$ car, la raie d'élongation de la liaison $\mathrm{CN}^{-}$ $\left(2088 \mathrm{~cm}^{-1}\right)$ pour $\mathrm{NaCN}$ étant totalement polarisée, on a :

$$
I_{x y, x y} \equiv 0
$$

pour cette raie, les relations (1) se réduisent à leur premier terme de membre de droite.

On en déduit que :

$$
\frac{\alpha}{1-\alpha}=R=\frac{I_{\mathrm{CN}^{-}}^{\mathrm{HV}}}{I_{\mathrm{CN}^{-}}^{\mathrm{VV}}}, \quad \frac{\beta}{1-\beta}=r=\frac{I_{\mathrm{CN}^{-}}^{\mathrm{VH}}}{I_{\mathrm{CN}^{-}}^{\mathrm{VV}}}
$$

et que

$$
\begin{aligned}
& I_{x x, x x} \cdot I_{0}^{\mathrm{V}}=\frac{1}{1-r}\left[I^{\mathrm{VV}}-r I^{\mathrm{VH}}+\left(I^{\mathrm{VV}}-I^{\mathrm{VH}}\right)(1+r) R\right] \\
& I_{x y, x y} \cdot I_{0}^{\mathrm{V}}=\frac{1}{1-r}\left[I^{\mathrm{VH}}-r I^{\mathrm{Vv}}\right]
\end{aligned}
$$

\section{Annexe II}

Corrélation entre les orientations des ions $\mathbf{C N}^{-}$en phase plastique. - Les corrélations sont prises entre les plus proches voisins qui, par exemple, sont situés en $0,0,0$; et $1 / 2,1 / 2,0$, puisque le cristal est cubique faces centrées. La fonction de probabilité donnant la probabilité de trouver un ion $\mathrm{CN}^{-}$noté $\mathrm{L}$ dans la direction $\Omega^{\mathrm{L}}$ à un instant $t$ et de trouver celui noté $\mathrm{L}^{\prime}$ au même instant dans la direction $\Omega^{\mathrm{L}^{\prime}} \mathrm{s}^{\prime e ́ c r i t}$ :

$$
P\left(\Omega^{\mathrm{L}}, \Omega^{\mathrm{L}^{\prime}}\right)=\sum_{\substack{l_{1} l_{2} \\ m_{1} m_{2}}} \gamma_{l_{1} l_{2}}^{m_{1} m_{2}} Y_{l_{1}}^{m_{1}}\left(\Omega^{\mathrm{L}}\right) Y_{l_{2}}^{m_{2}}\left(\Omega^{\mathrm{L}^{\prime}}\right)
$$

Les propriétés de la liaison entre ces deux ions permettent de réduire le nombre des coefficients. D'autre part, nous avons montré que les spectres de diffusion Raman sont exprimables en fonction des harmoniques sphériques de $J=2$. Il est donc suffisant de rechercher l'influence des propriétés de symétrie sur les harmoniques de rang 2 .

La symétrie de la liaison étant $\mathrm{C}_{2 \mathrm{v}}$, on en déduit des combinaisons linéaires dont les propriétés de symétrie sont les mêmes que celles des représentations irréductibles du groupe, soit :

$$
\begin{aligned}
& \text { pour } \mathrm{A}_{1} \quad K_{1}^{\mathbf{A}_{1}}=Y_{2}^{0}(\theta, \varphi) \quad \text { pour } \mathrm{A}_{2} \quad K^{\mathbf{A}_{2}}=\frac{1}{\sqrt{2}}\left(Y_{2}^{1}+i Y_{2}^{-1}\right) \\
& K_{2}^{\mathrm{A}_{1}}=\frac{1}{\sqrt{2}}\left(Y_{2}^{2}-Y_{2}^{-2}\right) \\
& \mathrm{B}_{1} \quad K^{\mathrm{B}_{1}}=\frac{1}{\sqrt{2}}\left(Y_{2}^{2}+Y_{2}^{-2}\right) \quad \mathrm{B}_{2} \quad K^{\mathrm{B}_{2}}=\frac{1}{\sqrt{2}}\left(Y_{2}^{1}-i Y_{2}^{-1}\right) .
\end{aligned}
$$

La fonction $P\left(\Omega^{\mathrm{L}}, \Omega^{\mathrm{L}^{\prime}}\right)$ devant nécessairement être de symétrie $\mathrm{A}_{1}$, le développement de la fonction pourra s'écrire à l'aide de 6 coefficients (on s'est limité aux interactions entre ions voisins)

$$
\begin{aligned}
P\left(\Omega^{\mathrm{L}}, \Omega^{\mathrm{L}^{\prime}}\right)= & \gamma_{1} K_{1}^{\mathrm{A}_{1}}\left(\Omega^{\mathrm{L}}\right) K_{1}^{\mathrm{A}_{1}}\left(\Omega^{\mathrm{L}^{\prime}}\right)+\gamma_{2} K_{2}^{\mathrm{A}_{1}}\left(\Omega^{\mathrm{L}}\right) K_{2}^{\mathrm{A}_{1}}\left(\Omega^{\mathrm{L}^{\prime}}\right) \\
& +\gamma_{3} K_{1}^{\mathrm{A}_{1}}\left(\Omega^{\mathrm{L}}\right) K_{2}^{\mathrm{A}_{1}}\left(\Omega^{\mathrm{L}^{\prime}}\right)+\gamma_{4} K^{\mathrm{A}_{2}}\left(\Omega^{\mathrm{L}^{2}}\right) K^{\mathrm{A}_{2}}\left(\Omega^{\mathrm{L}^{\prime}}\right) \\
& +\gamma_{5} K^{\mathbf{B}_{1}}\left(\Omega^{\mathrm{L}^{\mathrm{L}}}\right) K^{\mathbf{B}_{1}}\left(\Omega^{\mathrm{L}^{\prime}}\right)+\gamma_{6} K^{\mathrm{B}_{2}}\left(\Omega^{\mathrm{L}^{\mathrm{L}}}\right) K^{\mathbf{B}_{2}}\left(\Omega^{\mathrm{L}^{\prime}}\right) .
\end{aligned}
$$


Les deux ions sont équivalents, on doit donc symétriser les expressions du type :

$$
K^{\alpha}\left(\Omega^{\mathrm{L}}\right) K^{\alpha}\left(\Omega^{\mathrm{L}^{\prime}}\right) \rightarrow\left(K^{\alpha}\left(\Omega^{\mathrm{L}}\right) K^{\alpha}\left(\Omega^{\mathrm{L}^{\prime}}\right)+K^{\alpha}\left(\Omega^{\mathrm{L}^{\prime}}\right) K^{\alpha}\left(\Omega^{\mathrm{L}}\right)\right) .
$$

On en déduit aisément les valeurs des corrélations dans les spectres intégrés :

$$
\int \mathrm{E}_{\mathbf{g}}(\omega) \mathrm{d} \omega \sim \gamma_{1} \quad \text { et } \quad \int \mathrm{F}_{2 \mathrm{~g}}(\omega) \mathrm{d} \omega \sim\left(\gamma_{4}-\gamma_{6}\right)
$$

\section{References}

[1] O'Reilly, D. E., J. Chem. Phys. 58 (1973) 3032.

[2] O'Reilly, D. E., Peterson, E. M., Scheie, C. E. and KadaBA, P. K., J. Chem. Phys. 58 (1973) 3018.

[3] Price, D. L., Rowe, J. M., Rush, J. J., Prince, E., Hinks, D. G. et Susman, S., J. Chem. Phys. 56 (1972) 3697.

[4] Rowe, J. M., Hinks, D. G., Price, D. L., Susman, S. et Rush, J. J., J. Chem. Phys. 58 (1973) 2039.

[5] Daubert, J., Knorr, K., Dultz, W., Jex, H. et Currat, R., J. Phys. C 9 (1976) L-389.

[6] Winchester, L. W., Ph. D. University of Illinois (1975).

[7] Mathieu, J. P., Bull. Soc. Fr. Minéral. Cristallogr. LXXIX (1956) 96.

[8] Dultz, W., Solid State Commun. 15 (1974) 595.

[9] Callender, R. et Pershan, P. S., Phys. Rev. A 2 (1970) 672.

[10] Callender, R. et Pershan, P. S., Phys. Rev. Lett. 23 (1969) 947.

[11] Fontaine, D., Thèse Paris (1978).

[12] Fontaine, D. et Pick, R. M., Proc. 5th Int. Conf. Raman Spectr., edited by Schmid, E. D. (Hansferdinand Schulz Verlag, Freiburg im Breisgau) 1976, p. 606.

[13] Fontaine, D., Pick, R. M. et Yvinec, M., Solid State Commun. 21 (1977) 1095.

[14] Boissier, M., Vacher, R., Fontaine, D. et Pick, R. M., J. Physique 39 (1978) 205.

[15] Rehwald, W., Sandercok, J. R. et Rossinelli, M., Phys. Status Solidi (a) 42 (1977) 699.
[16] Structure Reports, vol. 9, p. 138, General editor A. J. C. Wilson by N. V. A. Oosthoek's uitgevers MIJ, Utrecht.

[17] Pauling, L., Phys. Rev. 36 (1930) 430.

[18] Frenkel, J., Acta Phys. Chem. URSS 3 (1939) 201.

[19] Couzi, M., Sokoloff, J. B. et Perry, C. H., J. Chem. Phys. 58 (1973) 2965.

[20] PICK, R. M., Physics of impurity center in crystals (Tallin, URSS) 1972.

[21] Rowe, J. M., Rush, J. J., Vegaletos, N., Price, D. L., Hinks, D. G. et Susman, S., J. Chem. Phys. 62 (1975) 4551.

[22] Rowe, J. M., Rush, J. J., Chesser, N. J., Michel, K. H. et NAudTs, J., Phys. Rev. Lett. 40 (1978) 455.

[23] Haussuhl, S., Solid State Commun. 13 (1973) 147.

[24] Michel, K. H. et Naudis, J., J. Chem. Phys. 67 (1977) 547.

[25] Rotenberg, M., Metropolis, N., Bivins, R. et Wooten, J. K. Jr., The $3 j$ and $6 j$ symbols (Crosby Lokwood and Son LTD London) 1959.

[26] Mathieu, J. P., Spectres de vibration et symétrie des molécules et des cristaux (Herman et Cie) 1945, p. 240.

[27] Sandercock, J. R., Communication privée.

[28] Kastler, A. et Rousset, A., J. Physique Radium 2 (1941) 49.

[29] TURell, J., Infrared and Raman Spectra of Crystals (Academic Press, London) 1972, p. 227.

[30] Damien, J. C., Thèse, Lille (1978). 